\title{
New Building Block for Polyhydroxylated Piperidine: Total synthesis of 1,6-Dideoxynojirimycin
}

Rajesh Rengasamy, Marcus J. Curtis-Long, ${ }^{\dagger}$ Woo Duck Seo, Seong Hun Jeong, Ill-Yun Jeong, ${ }^{\ddagger}$ and Ki Hun Park*

Division of Applied Life Science (BK21 program), EB-NCRC, Gyeongsang National University, Jinju 660-701, South Korea, and12 New Road, Nafferton, Driffield, East Yorkshire, YO25 4JP. Radiation Research Division, Korea Atomic Energy Research Institute, Jeongeup, 580-185, South Korea.

\section{SUPPORTING INFORMATION}

1. General Procedures

S2

2. Synthesis and Spectroscopic Data

S2

3. NMR and Mass Spectrum

S5 


\section{$\underline{\text { General Procedures }}$}

All NMR spectra were obtained on a $300 \mathrm{MHz}$ spectrometer. 2D experiments NOSEY and COSY were used to assist analysis of 1D spectra obtained on a $500 \mathrm{MHz}$ spectrometer. All NMR experiments were run using standard routines. Flash column chromatography was performed using pre-packed silica gel columns. Thin layer chromatography was performed on commercially available glass-backed silica gel plates. Optical Rotations were measured using the Na-D line. All starting materials were dried in a vacuum for one day before use. All non-aqueous reactions were carried out under inert $\mathrm{N}_{2}$ atmosphere, and reaction solvents were distilled according to standard procedures unless stated otherwise.

(S)-methyl 2-(tert-butoxycarbonylamino)propanoate (4).Prepared a suspension of L-alanine (5 g, $56.12 \mathrm{mmol})$ in $(82.5 \mathrm{~mL})$ of $\mathrm{MeOH}$. Stirred, cooled to $0{ }^{\circ} \mathrm{C}$. Carefully added $(9 \mathrm{~mL}, 123.46 \mathrm{mmol})$ of $\mathrm{SOCl}_{2}$ dropwise. The reaction mixture was then warmed to $40{ }^{\circ} \mathrm{C}$. After $3.5 \mathrm{~h}$, the reaction mixture was then cooled to rt. The solvent was removed by rotary evaporation to afford alanine ester hydrochloride (7.75 $\mathrm{g}, 99 \%)$ as a white solid. Dissolved (7 $\mathrm{g}, 50.15 \mathrm{mmol})$ of alanine ester hydrochloride in 1:1 mixture of $\mathrm{THF} / \mathrm{H}_{2} \mathrm{O}(167 \mathrm{~mL}),, \mathrm{NaHCO}_{3}$ and $\mathrm{Boc}_{2} \mathrm{O}$ were added consecutively stirred at room temperature for $7 \mathrm{~h}$. The turbid solution was extracted with ether. The aqueous layer was acidified to $\mathrm{pH}$ = 4-5 by careful addition of sat. citric acid at $0{ }^{\circ} \mathrm{C}$ and then extracted with $\mathrm{CH}_{2} \mathrm{Cl}_{2}$. The combined organic phase was dried Na2SO4 and evaporated. The product was isolated by flash column chromatography (hexane: EtOAc $=80: 20)$ to give $4(9.3 \mathrm{~g}, 92 \%)$ as an yellow oil; $[\alpha]^{20}{ }_{\mathrm{D}}-3.4(\mathrm{c} 1$, $\left.\mathrm{CHCl}_{3}\right) ;{ }^{1} \mathrm{H} \mathrm{NMR}\left(300 \mathrm{MHz} ; \mathrm{CDCl}_{3}\right) \delta 1.34(3 \mathrm{H}, \mathrm{d}, \mathrm{J}=7.2 \mathrm{~Hz}), 1.39(9 \mathrm{H}, \mathrm{s}), 3.69(3 \mathrm{H}, \mathrm{s}), 4.26(1 \mathrm{H}$, m), $5.26(1 \mathrm{H}$, brs, $\mathrm{NH}) ;{ }^{13} \mathrm{C}$ NMR $\left(300 \mathrm{MHz} ; \mathrm{CDCl}_{3}\right) \delta 18.4,28.2,49.0,52.1,79.6,155.0,173.7$; EIMS (m/z) $203\left(\mathrm{M}^{+}\right)$; HRMS calcd for $\mathrm{C}_{9} \mathrm{H}_{17} \mathrm{NO}_{4}\left(\mathrm{M}^{+}\right)$203.1158, found 203.1159.

(S)-tert-butyl 1-hydroxypropan-2-ylcarbamate (5). To a suspension of (2.5 g, 66.40 $\mathrm{mmol}) \mathrm{LiAlH}_{4}$ in $(128 \mathrm{~mL})$ of dry THF. Stirred, cooled to $0{ }^{\circ} \mathrm{C}$. Added a solution of 4 (9 g, $\left.44.28 \mathrm{mmol}\right)$ in $(20 \mathrm{~mL})$ of dry THF. After addition the reaction mixture was then brought to $\mathrm{rt}$ and quenched by the sequential addition 
of $4.5 \mathrm{~mL}$ of $\mathrm{H}_{2} \mathrm{O}, 4.5 \mathrm{~mL}$ of $15 \%$ aq. $\mathrm{NaOH}$ and $12.5 \mathrm{~mL}$ of $\mathrm{H}_{2} \mathrm{O}$. The mixture was then poured into $(150 \mathrm{~mL})$ of $\mathrm{Et}_{2} \mathrm{O}$ and stirred for $30 \mathrm{~min}$. The insoluble material was removed by filtration through Celite and the solvent was removed from the filtrate by rotary evaporation dried over $\mathrm{Na}_{2} \mathrm{SO}_{4}$. The product was isolated by flash column chromatography on silica gel using (hexane: EtOAc $=70: 30$ ) to give $5(7.50 \mathrm{~g}, 97 \%)$ as white solid; $\mathrm{Mp} 59-61{ }^{\circ} \mathrm{C} ;[\alpha]^{20}{ }_{\mathrm{D}}-9.3\left(\mathrm{c} 1, \mathrm{CHCl}_{3}\right) ;{ }^{1} \mathrm{H} \mathrm{NMR}(300 \mathrm{MHz}$; $\left.\mathrm{CDCl}_{3}\right) \delta 1.12(3 \mathrm{H}, \mathrm{d}, \mathrm{J}=6.7 \mathrm{~Hz}), 1.39(9 \mathrm{H}, \mathrm{s}), 3.48(2 \mathrm{H}, \mathrm{m}), 3.57(1 \mathrm{H}, \mathrm{m}), 5.0(1 \mathrm{H}, \mathrm{brs}, \mathrm{NH}) ;{ }^{13} \mathrm{C}$ $\operatorname{NMR}\left(300 \mathrm{MHz} ; \mathrm{CDCl}_{3}\right) \delta 17.2,28.3,48.4,66.5,79.5,156.3 ;$ EI-MS $(\mathrm{m} / \mathrm{z})\left(\mathrm{M}^{+}\right)$; HRMS calcd for $\mathrm{C}_{8} \mathrm{H}_{17} \mathrm{NO}_{3}\left(\mathrm{M}^{+}\right)$175.1208, found 175.1209

(S)-tert-butyl 1-(tert-butyldimethylsilyloxy)propan-2-ylcarbamate (6). Dissolved (6 g, 34.24 mmol) of 5 and $(6.99 \mathrm{~g}, 102.72 \mathrm{mmol})$ of imidazole in $(114 \mathrm{~mL})$ of $\mathrm{CH}_{2} \mathrm{Cl}_{2}$. Stirred at rt. Added (10.3 g, 68.48 mmol) of TBDMSCl and stirred overnight. After $16 \mathrm{~h}$, quenched with sat aq. $\mathrm{NH}_{4} \mathrm{Cl}$ Extracted with $\mathrm{CH}_{2} \mathrm{Cl}_{2}$. The organic layer was dried over $\mathrm{Na}_{2} \mathrm{SO}_{4}$, filtered and the solvent was removed by rotary evaporation. The product was isolated by flash chromatography on silica gel using (hexane: EtOAc = 80:20) to give $6(9.8 \mathrm{~g}, 99 \%)$ as colorless oil; $[\alpha]^{20}{ }_{\mathrm{D}}-20.2\left(\mathrm{c} 1, \mathrm{CHCl}_{3}\right) ;{ }^{1} \mathrm{H} \mathrm{NMR}\left(300 \mathrm{MHz} ; \mathrm{CDCl}_{3}\right) \delta$ $0.04(6 \mathrm{H}, \mathrm{s}), 0.89(9 \mathrm{H}, \mathrm{s}), 1.13(3 \mathrm{H}, \mathrm{d}, \mathrm{J}=6.6 \mathrm{~Hz}), 1.44(9 \mathrm{H}, \mathrm{s}), 3.52(2 \mathrm{H}, \mathrm{m}), 3.57(1 \mathrm{H}, \mathrm{brs}) ;{ }^{13} \mathrm{C}$ NMR $\left(300 \mathrm{MHz} ; \mathrm{CDCl}_{3}\right) \delta-8.0,15.0,15.7,23.3,25.8,45.1,63.6,76.4,152.8 ;$ FAB-MS obsd 290.2832, calcd $290.2151\left[(\mathrm{M}+\mathrm{H})^{+}, \mathrm{M}=\mathrm{C}_{14} \mathrm{H}_{31} \mathrm{NO}_{3} \mathrm{Si}\right]$.

(S)-tert-butyl allyl(1-(tert-butyldimethylsilyloxy)propan-2-yl)carbamate (7). To a stirred solution of $(0.99 \mathrm{~g}, 41.49 \mathrm{mmol}) \mathrm{NaH}$ in $(118 \mathrm{~mL})$ of dry DMF. Stirred, cooled to $0{ }^{\circ} \mathrm{C}$. Added as solution of $6(8 \mathrm{~g}$, $27.66 \mathrm{mmol})$ in $(20 \mathrm{~mL})$ of dry DMF dropwise. Stirred for $15 \mathrm{~min}$. Added $(7.69 \mathrm{~mL}, 63.60 \mathrm{mmol})$ of allylbromide and allowed the reaction mixture to slowly warm to room temperature. After $24 \mathrm{~h}$ the reaction was quenched with $\mathrm{H}_{2} \mathrm{O}$, sat. aq. $\mathrm{NH}_{4} \mathrm{Cl}$ and extracted with EtOAc. The organic layer was dried over $\mathrm{Na}_{2} \mathrm{SO}_{4}$, filtered and the solvent was removed by rotary evaporation. The product was purified by flash column chromatography (hexane: EtOAc $=90: 10)$ to give $7(6.7 \mathrm{~g}, 74 \%)$ as colorless oil; $[\alpha]^{20}{ }_{\mathrm{D}}^{-}$ 
$6.5\left(\mathrm{c} 1, \mathrm{CHCl}_{3}\right) ;{ }^{1} \mathrm{H} \mathrm{NMR}\left(300 \mathrm{MHz} ; \mathrm{CDCl}_{3}\right) \delta 0.06(6 \mathrm{H}, \mathrm{s}), 0.88(9 \mathrm{H}, \mathrm{s}), 1.13(3 \mathrm{H}, \mathrm{d}, \mathrm{J}=6.9 \mathrm{~Hz}), 1.44$ $(9 \mathrm{H}, \mathrm{s}), 3.55(2 \mathrm{H}, \mathrm{m}), 3.37(2 \mathrm{H}, \mathrm{brs}), 5.06(3 \mathrm{H}, \mathrm{m}), 5.66(1 \mathrm{H}, \mathrm{m}), 3.84(2 \mathrm{H}, \mathrm{m}) ;{ }^{13} \mathrm{C}$ NMR $(300 \mathrm{MHz}$; $\left.\mathrm{CDCl}_{3}\right) \delta 18.1,25.8,28.4,65.2,114.9,117.1,118.4,135.5,155.4$; FAB-MS obsd 330.2508, calcd $330.2464\left[(\mathrm{M}+\mathrm{H})^{+}, \mathrm{M}=\mathrm{C}_{17} \mathrm{H}_{35} \mathrm{NO}_{3} \mathrm{Si}\right]$.

tert-butyl allyl(S)-1-hydroxypropan-2-ylcarbamate (8). Dissolved $(5.5 \mathrm{~g}, 16.68 \mathrm{mmol})$ of $\mathbf{7}$ in (10 $\mathrm{mL}$ ) of THF. Added (33 mL, $33.36 \mathrm{mmol})$ of TBAF (1.0 M in THF). After $3 \mathrm{~h}$, quenched with sat. aq. $\mathrm{NH}_{4} \mathrm{Cl}$. Extracted with EtOAc. The organic layer was dried over $\mathrm{Na}_{2} \mathrm{SO}_{4}$, filtered and the solvent was removed by rotary evaporation. The product was isolated by flash column chromatography on silica gel using (hexane: $\mathrm{EtOAc}=70: 30)$ to give $8(3.38 \mathrm{~g}, 94 \%)$ as colorless oil; $[\alpha]^{20}{ }_{\mathrm{D}}+6.2\left(\mathrm{c} 1, \mathrm{CHCl}_{3}\right) ;{ }^{1} \mathrm{H}$ NMR (300 MHz; CDCl $) \delta 1.13(3 \mathrm{H}, \mathrm{d}, \mathrm{J}=6.9 \mathrm{~Hz}), 1.42(9 \mathrm{H}, \mathrm{s}), 3.56(2 \mathrm{H}, \mathrm{brs}), 3.72(2 \mathrm{H}, \mathrm{brs}), 3.99$ (1H, brs), $5.09(2 \mathrm{H}, \mathrm{m}), 5.82(1 \mathrm{H}, \mathrm{m}) ;{ }^{13} \mathrm{C}$ NMR $\left(300 \mathrm{MHz} ; \mathrm{CDCl}_{3}\right) \delta$ 14.7, 28.3, 46.9, 53.8, 65.0, 79.8, 115.7, 135.6, 156.2; EI-MS (m/z) $215\left(\mathrm{M}^{+}\right)$; HRMS calcd for $\mathrm{C}_{11} \mathrm{H}_{21} \mathrm{NO}_{3}\left(\mathrm{M}^{+}\right)$215.1521, found 215.1522. 


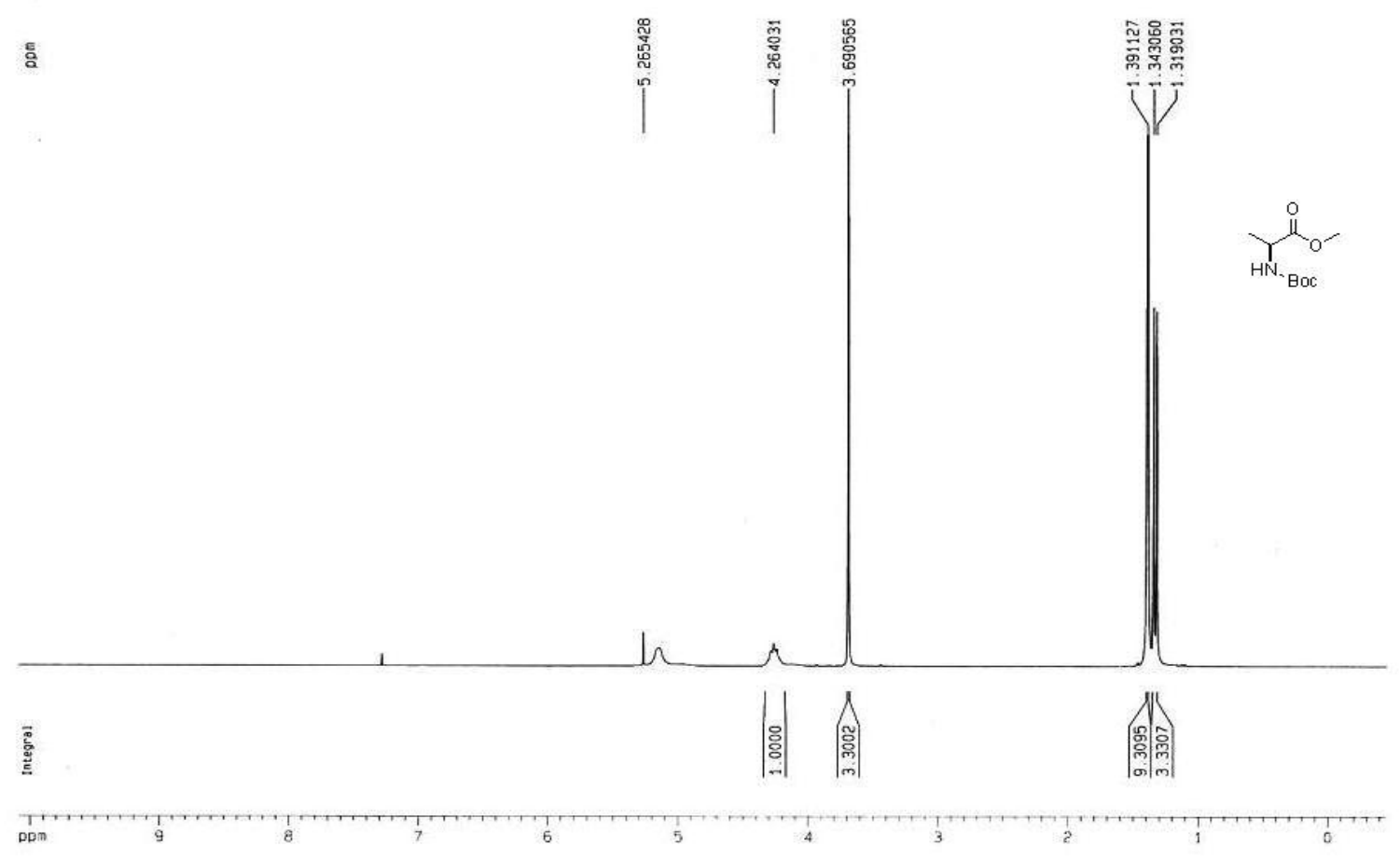

Spectra 1. ${ }^{1} \mathrm{H}$ NMR(300 MHz, $\left.\mathrm{CDCl}_{3}\right)$ of (S)-methyl 2-(tert-butoxycarbonylamino)propanoate (4)

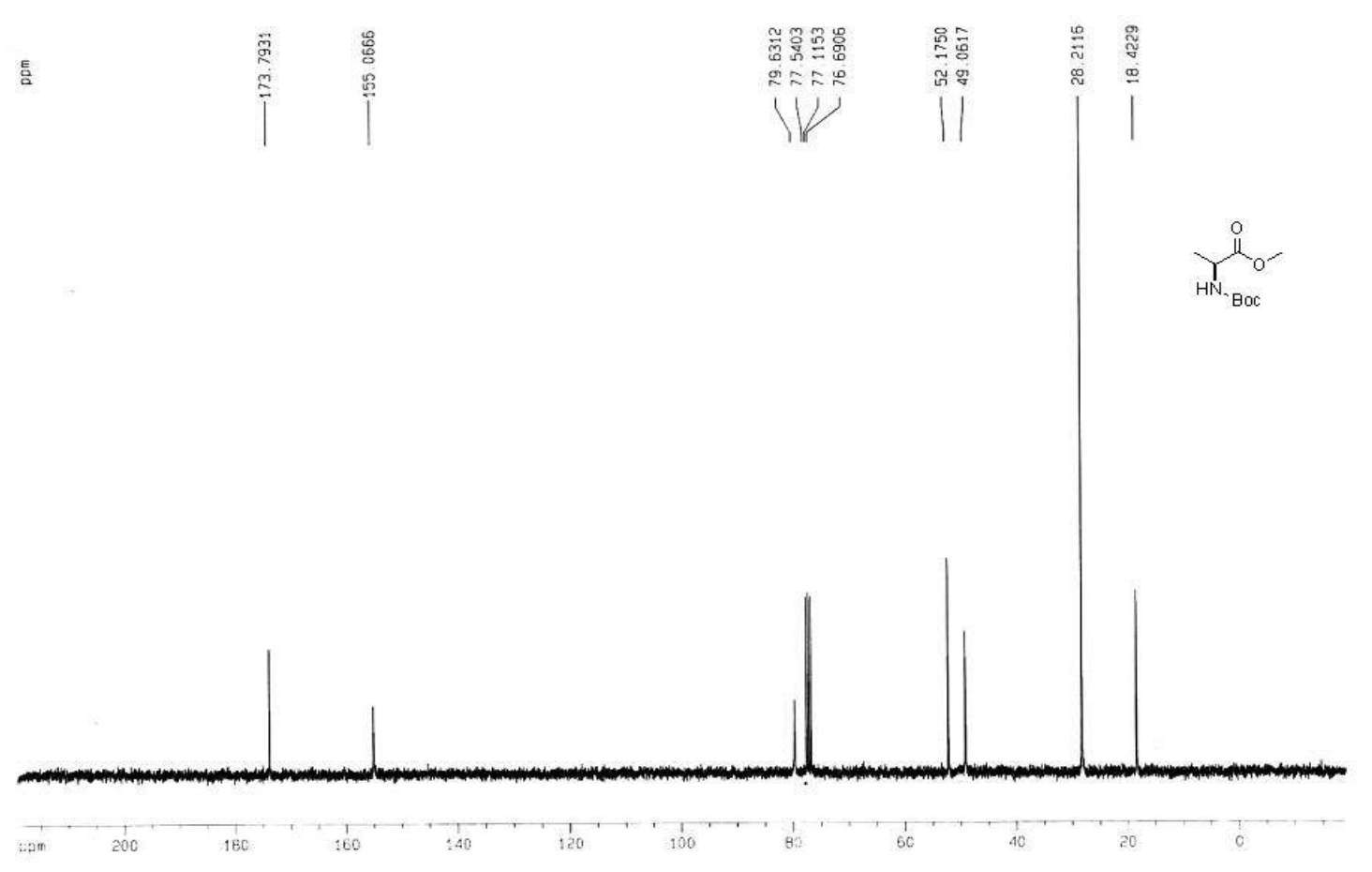

Spectra $2 .{ }^{13} \mathrm{C}$ NMR (300 MHz, $\left.\mathrm{CDCl}_{3}\right)$ of $(S)$-methyl 2-(tert-butoxycarbonylamino)propanoate (4) 


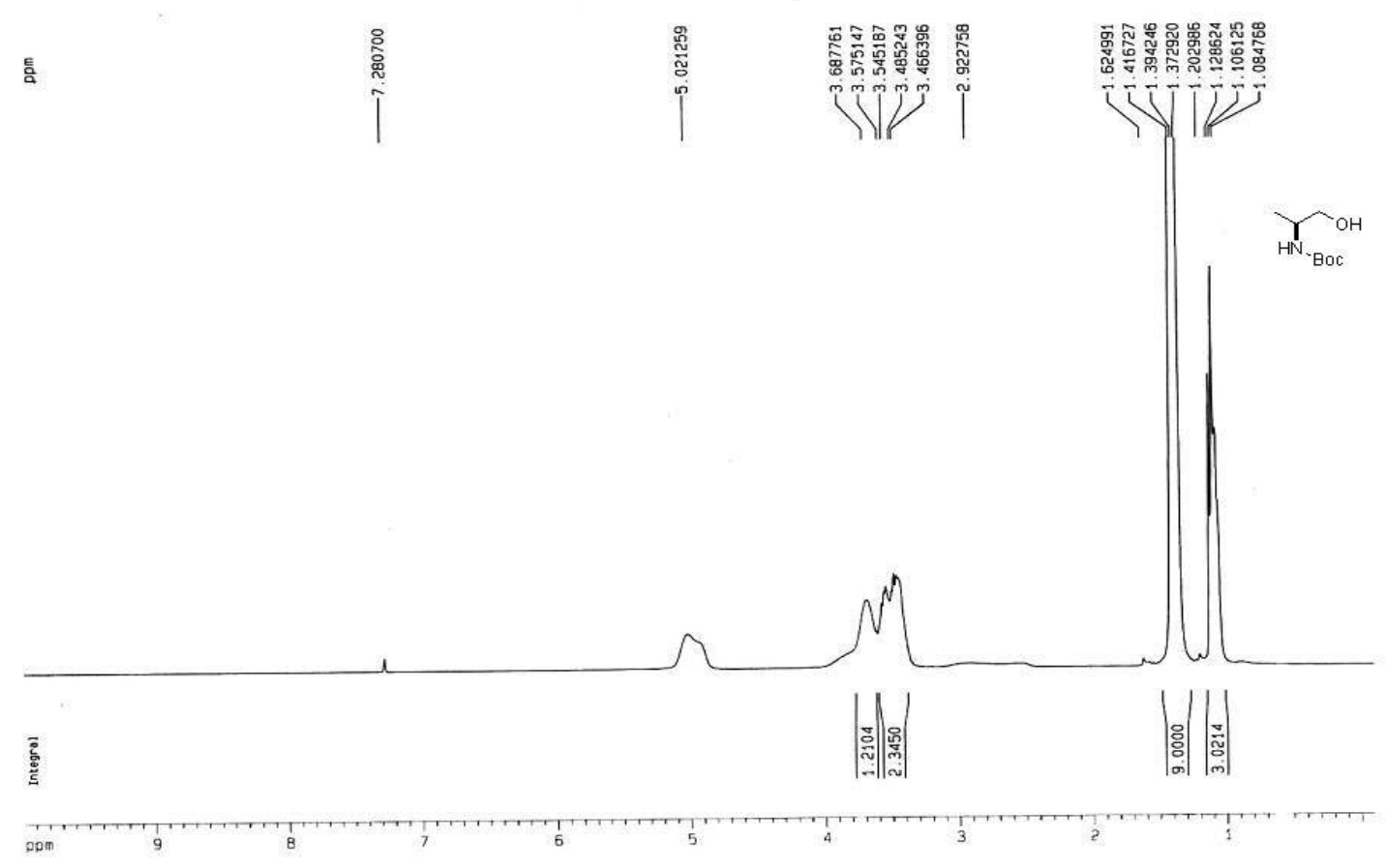

Spectra 3. ${ }^{1} \mathrm{H}$ NMR $\left(300 \mathrm{MHz}, \mathrm{CDCl}_{3}\right)$ of $(S)$-tert-butyl 1-hydroxypropan-2-ylcarbamate (5)

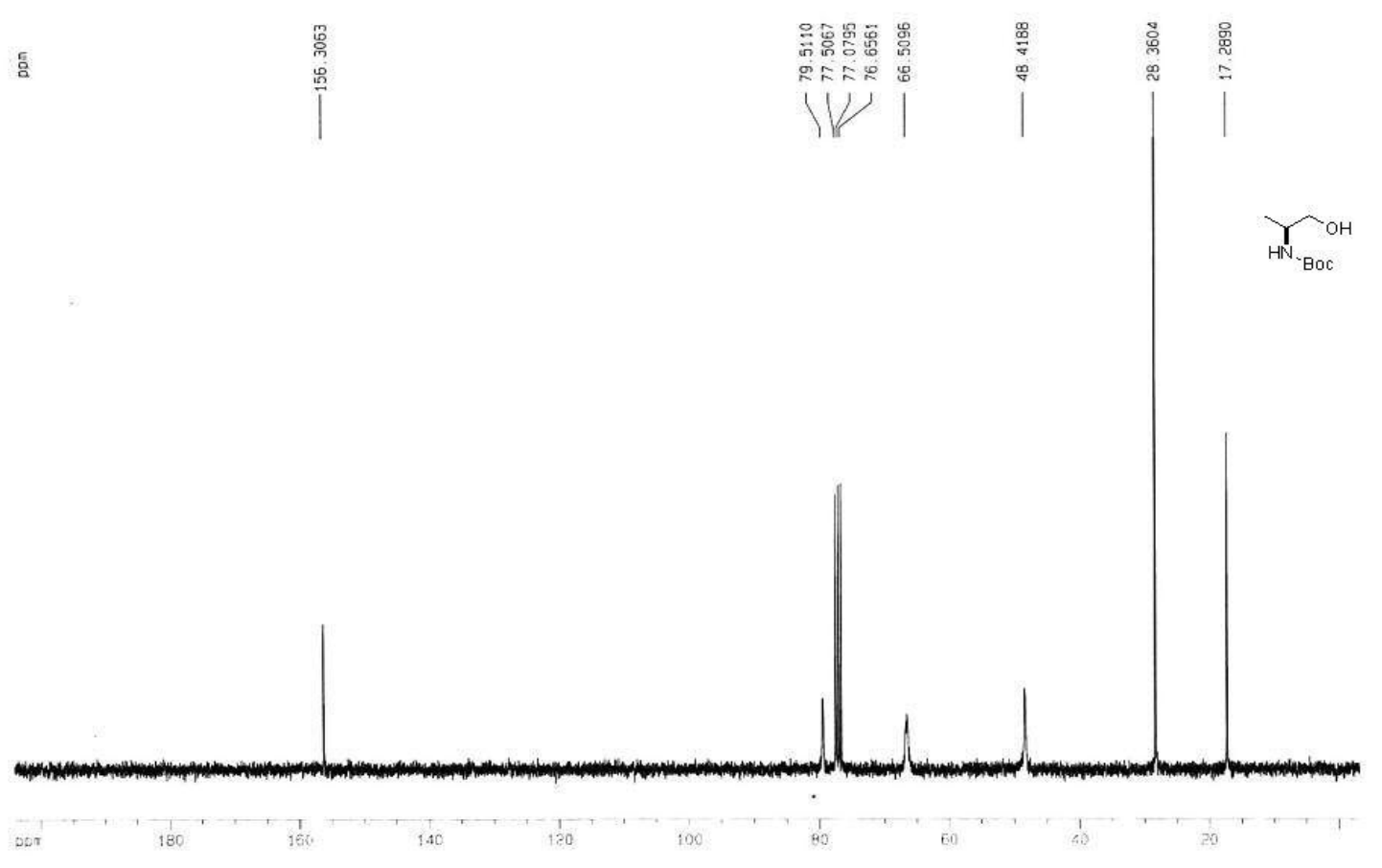

Spectra $4 .{ }^{13} \mathrm{C}$ NMR $\left(300 \mathrm{MHz}, \mathrm{CDCl}_{3}\right)$ of $(S)$-tert-butyl 1-hydroxypropan-2-ylcarbamate (5) 


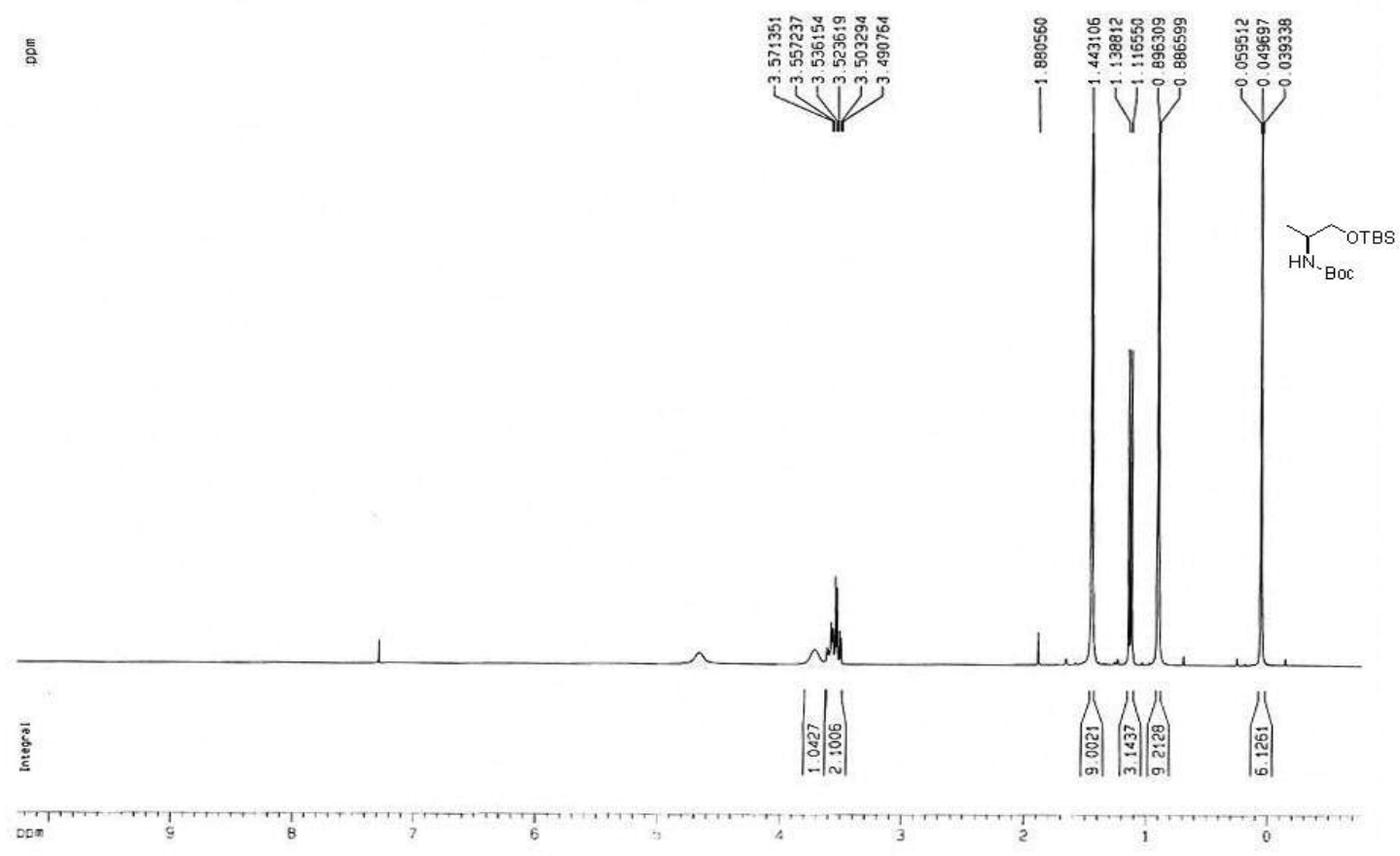

Spectra 5. ${ }^{1} \mathrm{H}$ NMR (300 $\left.\mathrm{MHz}, \mathrm{CDCl}_{3}\right)$ of $(S)$-tert-butyl 1-(tert-butyldimethylsilyloxy)propan-2ylcarbamate (6)

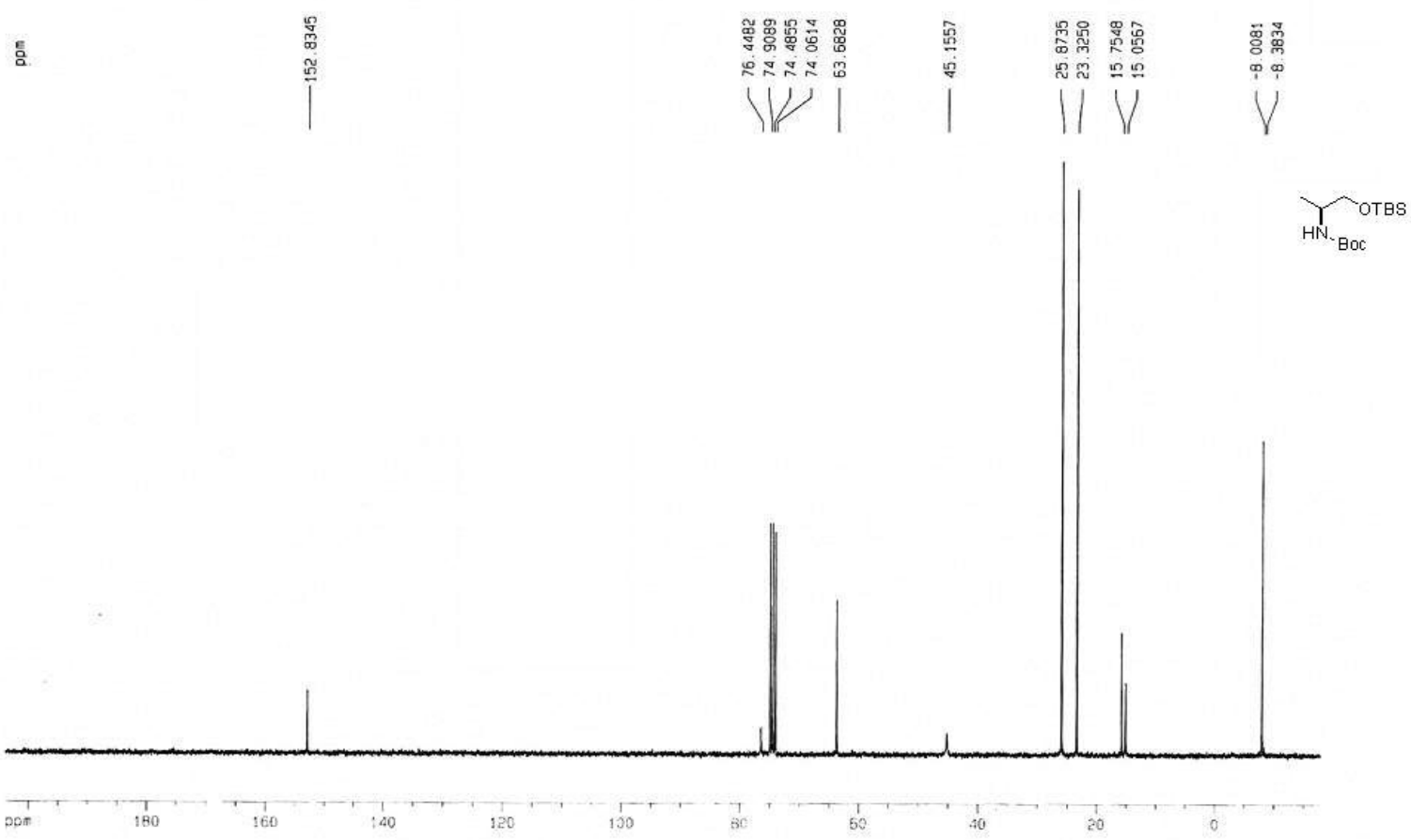

Spectra $6 .{ }^{13} \mathrm{C}$ NMR $\left(300 \mathrm{MHz}, \mathrm{CDCl}_{3}\right)$ of $(S)$-tert-butyl 1-(tert-butyldimethylsilyloxy)propan-2ylcarbamate (6) 


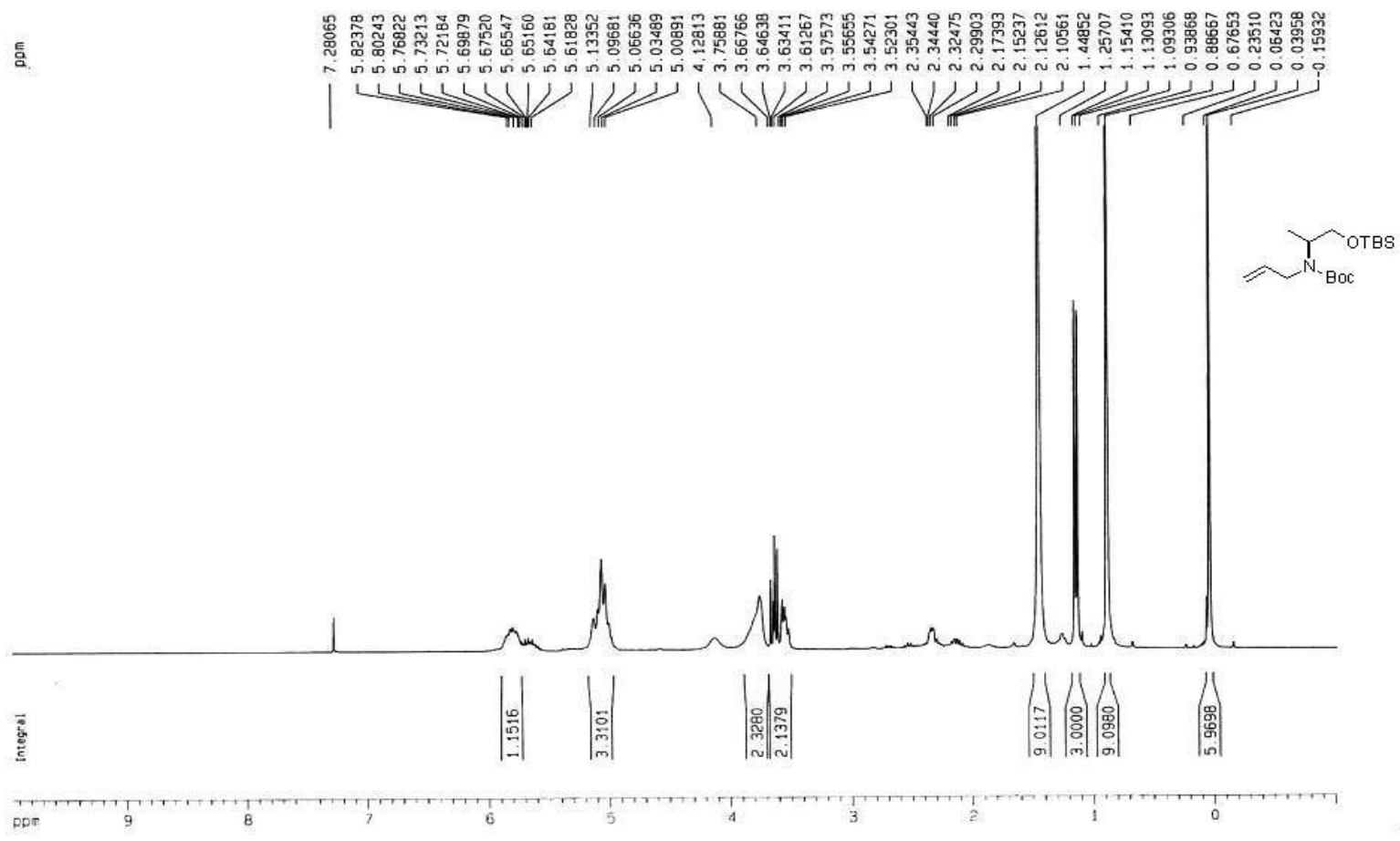

Spectra 7. ${ }^{1} \mathrm{H}$ NMR $\left(300 \mathrm{MHz}, \mathrm{CDCl}_{3}\right)$ of $(S)$-tert-butyl allyl(1-(tert-butyldimethylsilyloxy)propan2-yl)carbamate (7)

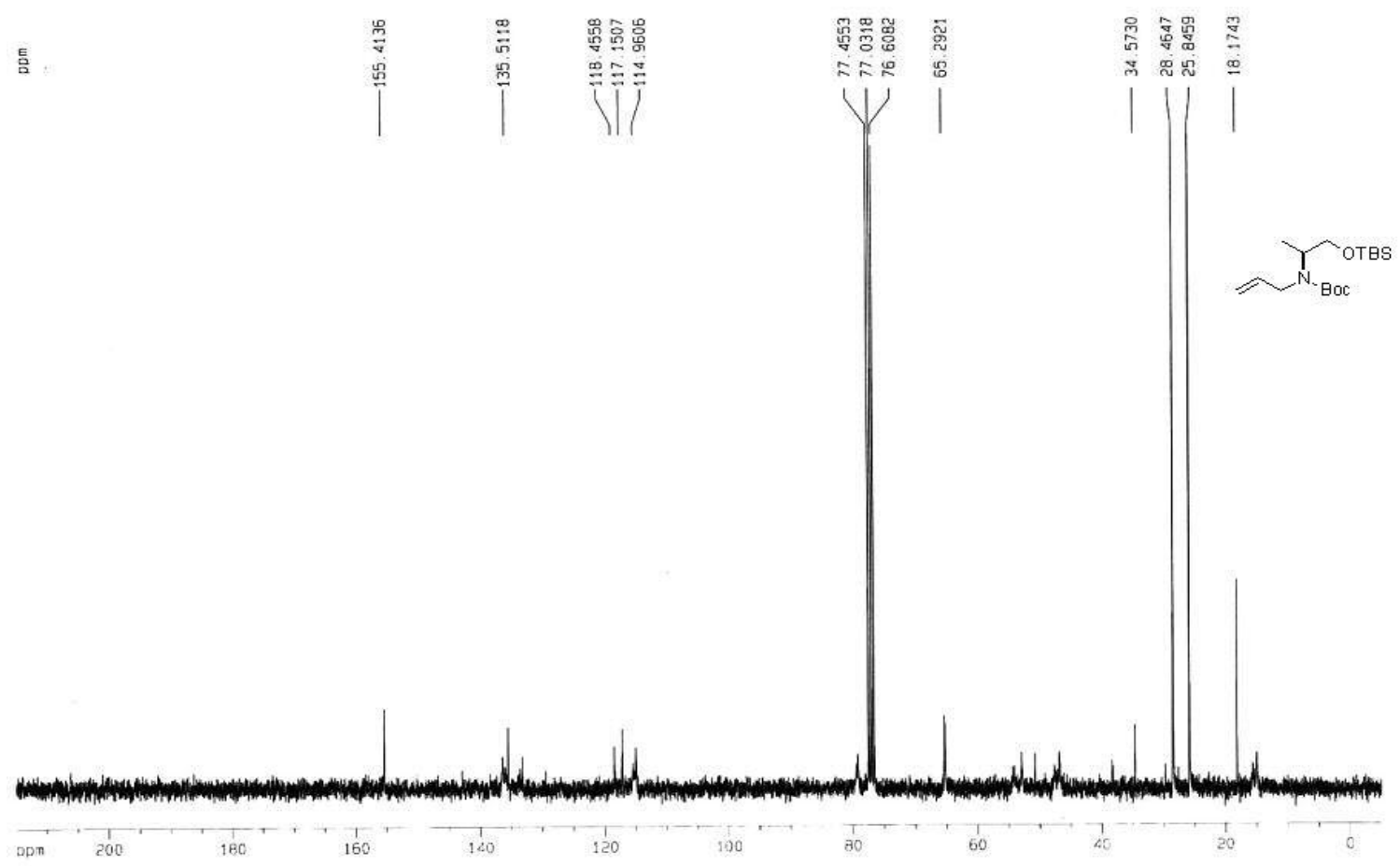

Spectra 8. ${ }^{13} \mathrm{C} \quad \mathrm{NMR} \quad\left(300 \quad \mathrm{MHz}, \quad \mathrm{CDCl}_{3}\right)$ of $(S)$-tert-butyl allyl [1-(tertbutyldimethylsilyloxy)propan-2-yl] carbamate (7) 


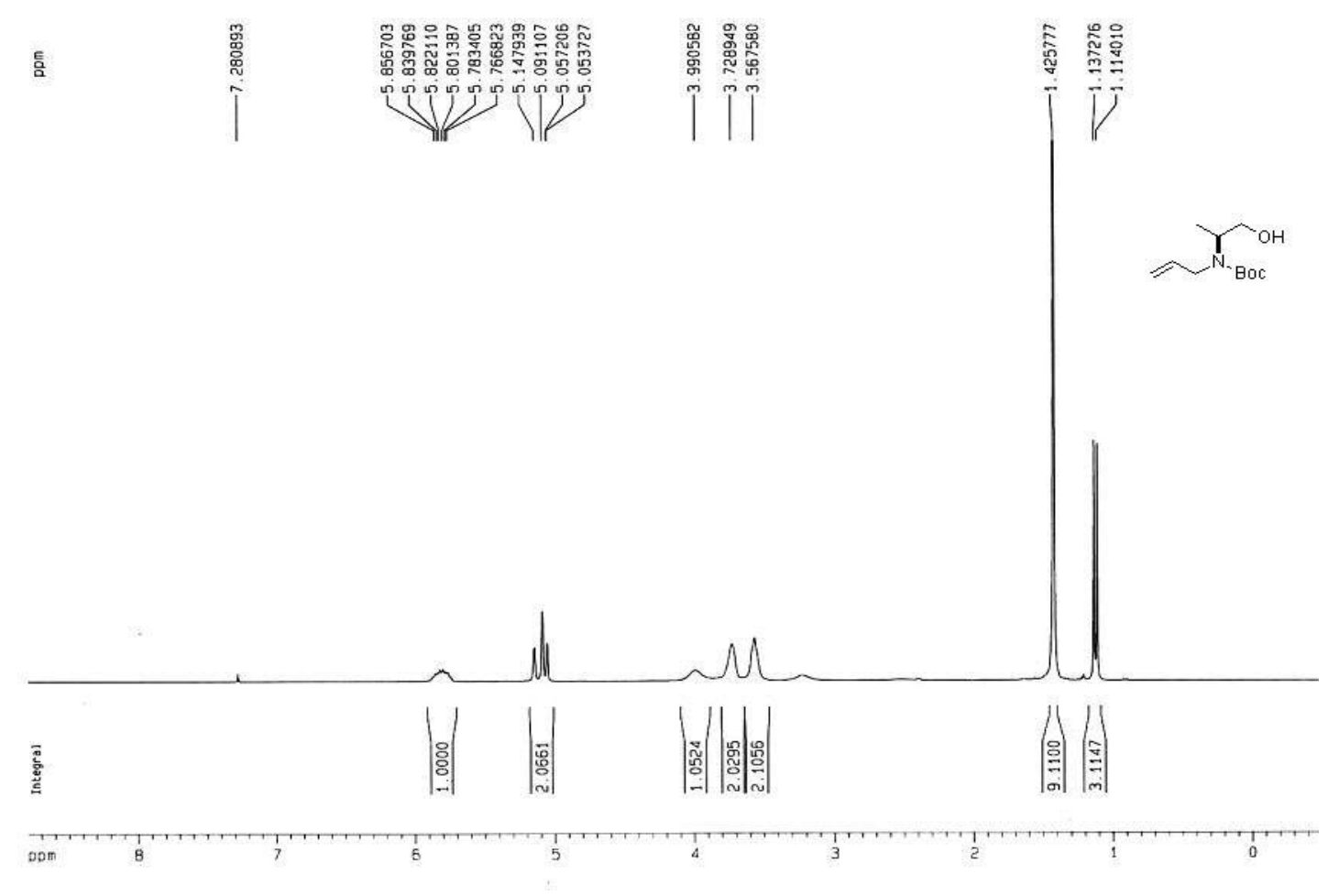

Spectra 9. ${ }^{1} \mathrm{H}$ NMR (300 MHz, $\left.\mathrm{CDCl}_{3}\right)$ of (S)-tert-butyl allyl(1-hydroxypropan-2-yl)carbamate (8)

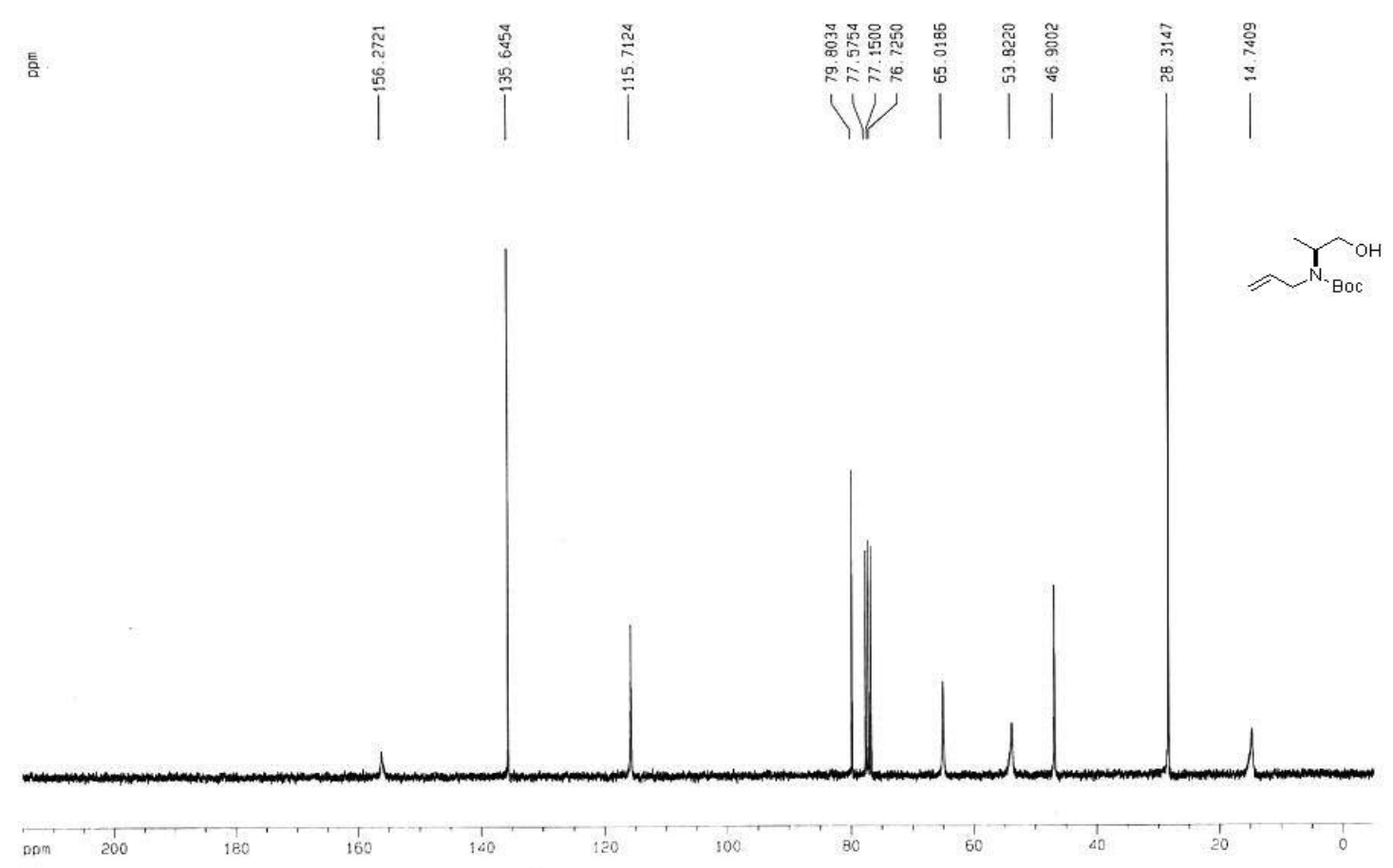

Spectra $10 .{ }^{13} \mathrm{C}$ NMR (300 $\left.\mathrm{MHz}, \mathrm{CDCl}_{3}\right)$ of $(S)$-tert-butyl allyl(1-hydroxypropan-2-yl)carbamate (8) 


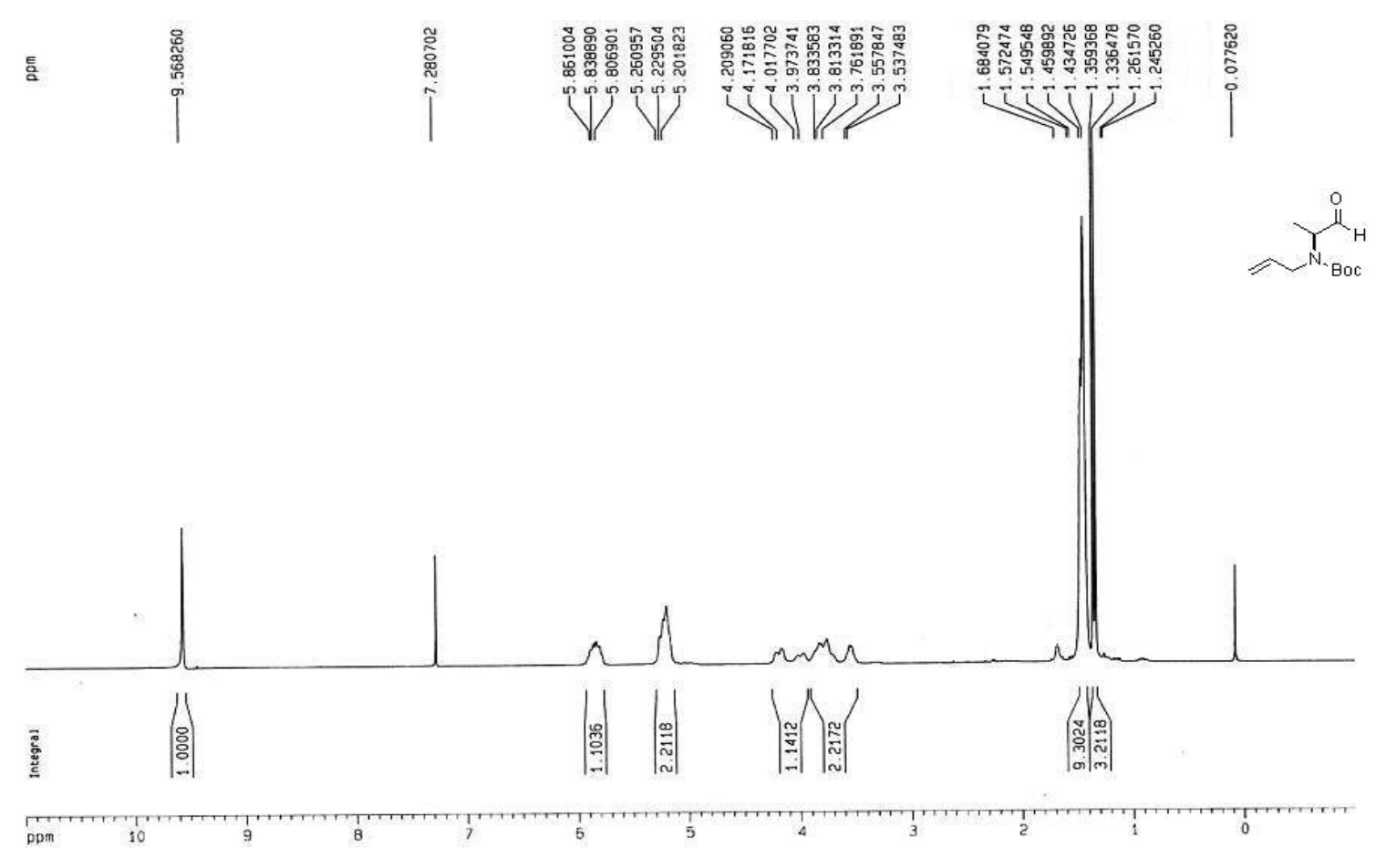

Spectra 11. ${ }^{1} \mathrm{H}$ NMR (300 MHz, $\mathrm{CDCl}_{3}$ ) of (S)-tert-butyl allyl(1-oxopropan-2-yl)carbamate (9)

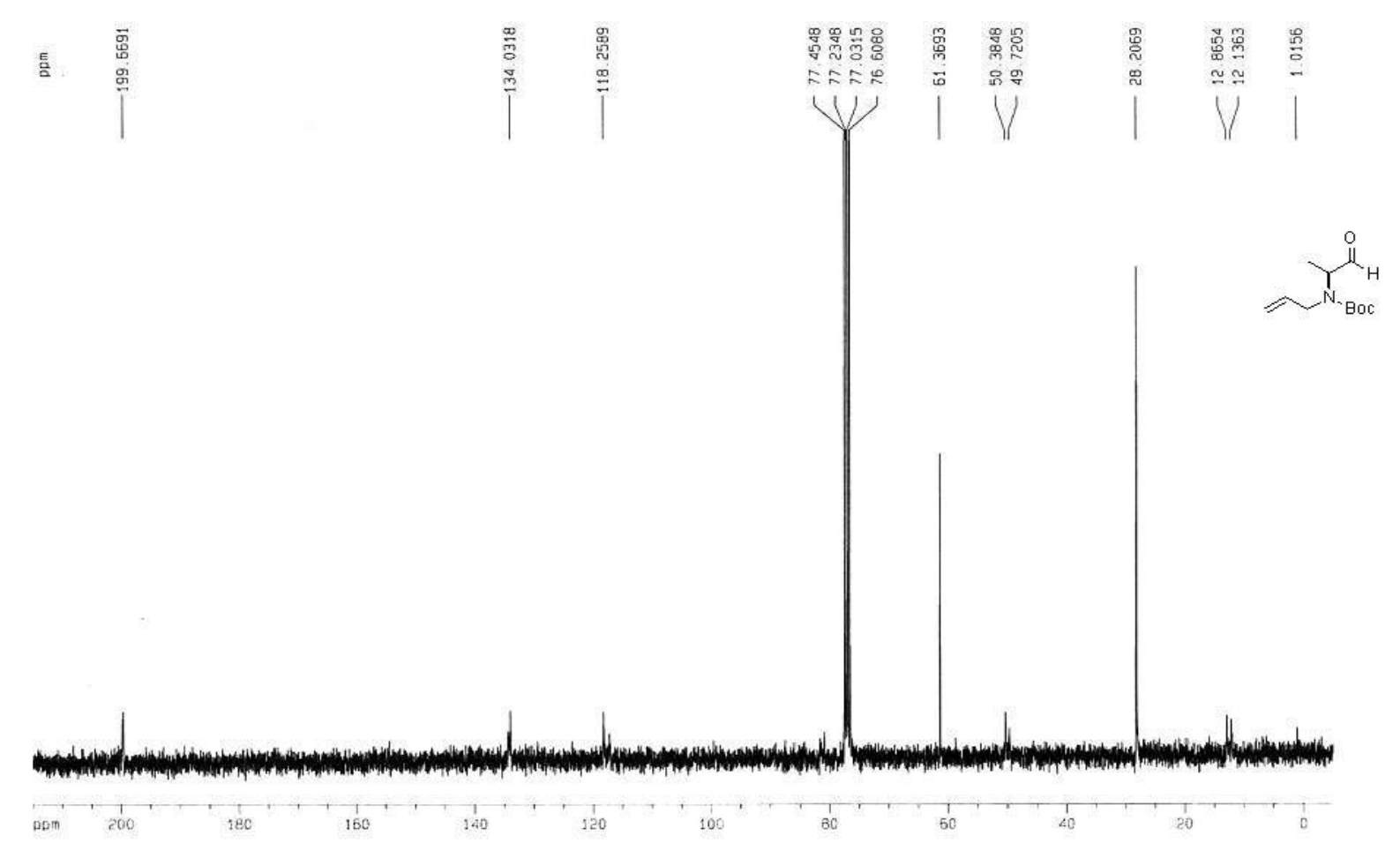

Spectra 12. ${ }^{13} \mathrm{C}$ NMR (300 MHz, $\left.\mathrm{CDCl}_{3}\right)$ of (S)-tert-butyl allyl(1-oxopropan-2-yl)carbamate (9) 


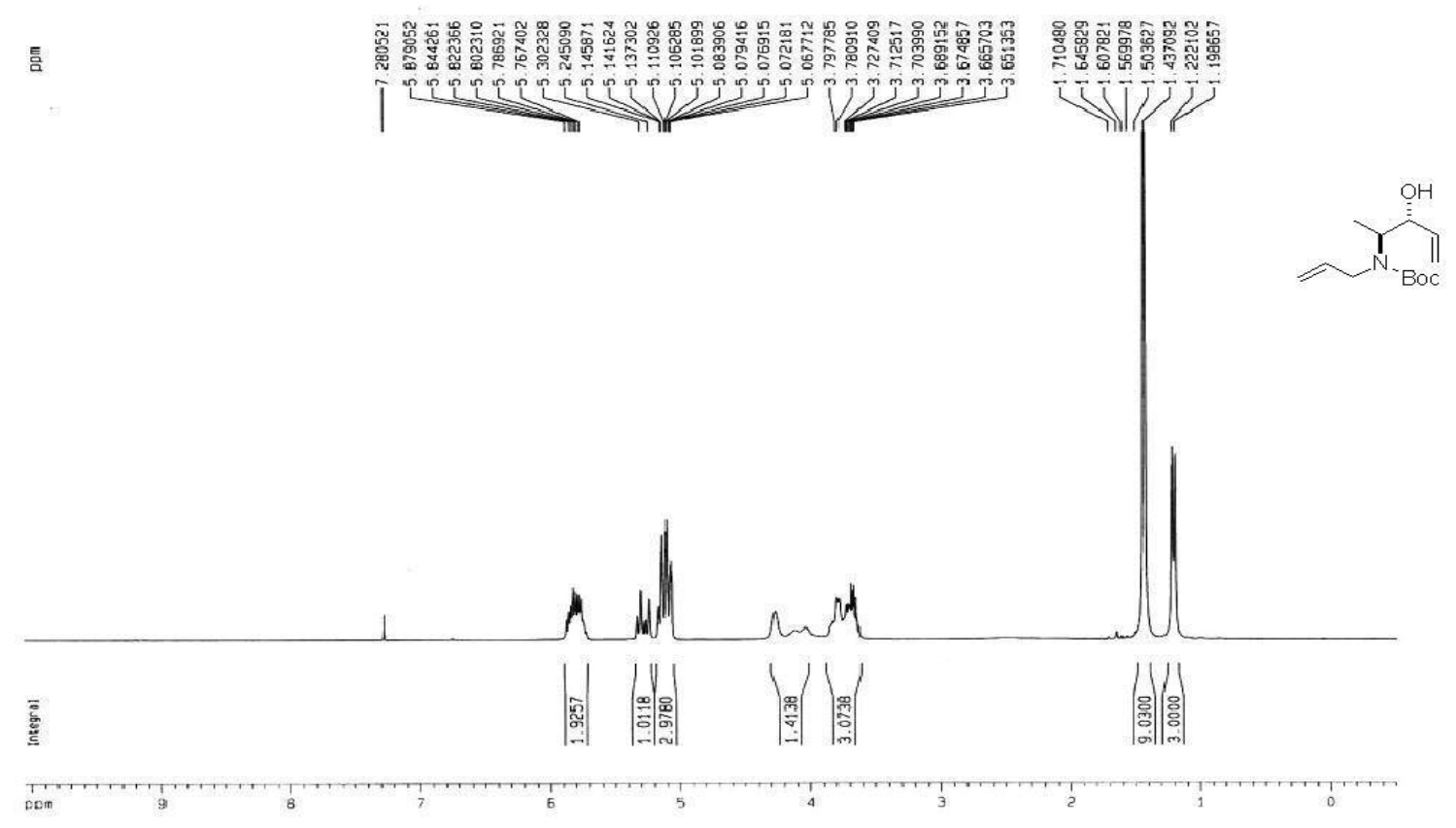

Spectra 13. ${ }^{1} \mathrm{H}$ NMR (300 $\left.\mathrm{MHz}, \mathrm{CDCl}_{3}\right)$ of tert-butyl allyl[(2S,3R)-3-hydroxypent-4-en-2-yl] carbamate (10)

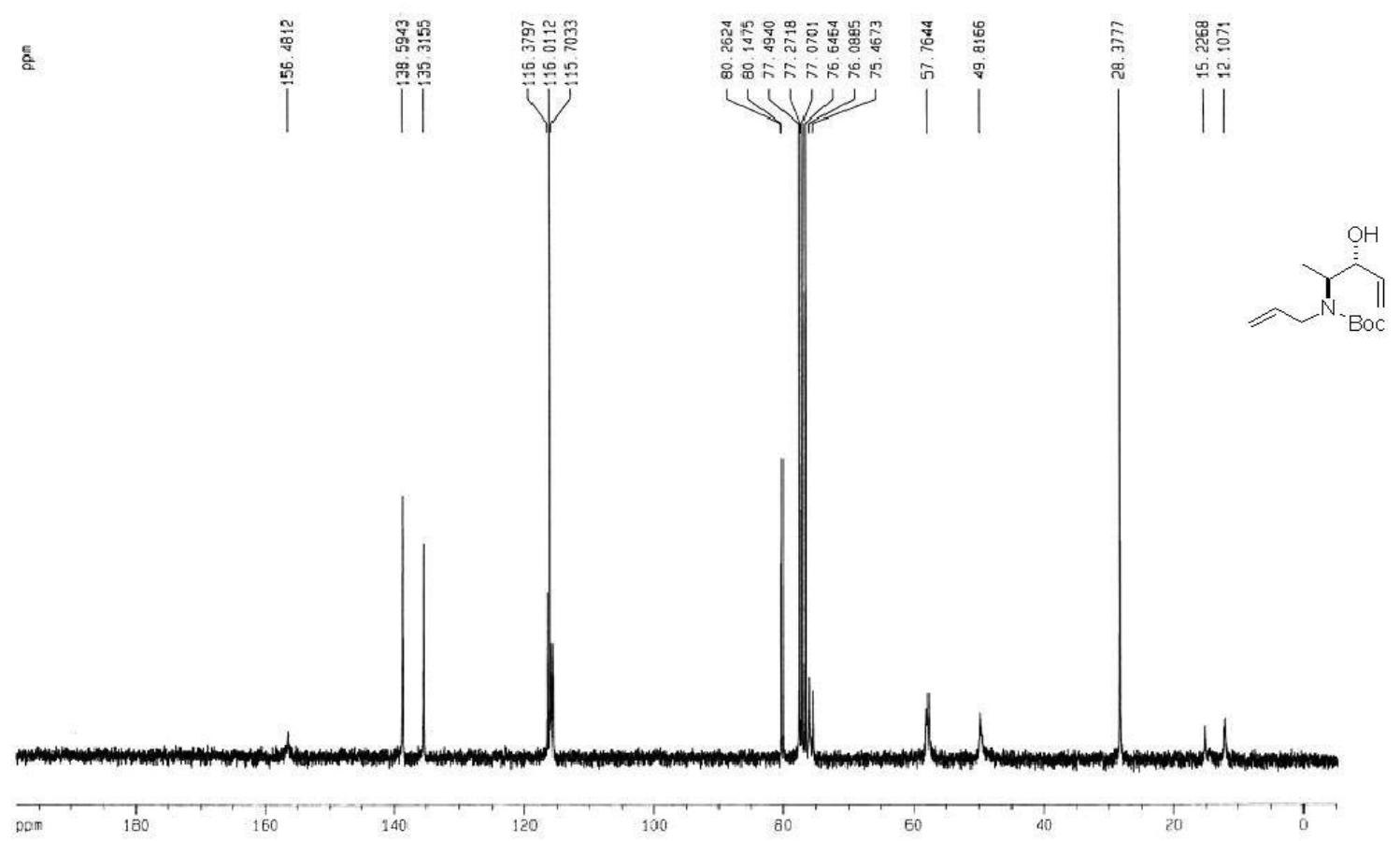

Spectra 14. ${ }^{13} \mathrm{C}$ NMR (300 $\left.\mathrm{MHz}, \mathrm{CDCl}_{3}\right)$ of tert-butyl allyl[(2S,3R)-3-hydroxypent-4-en-2-yl] carbamate (10) 


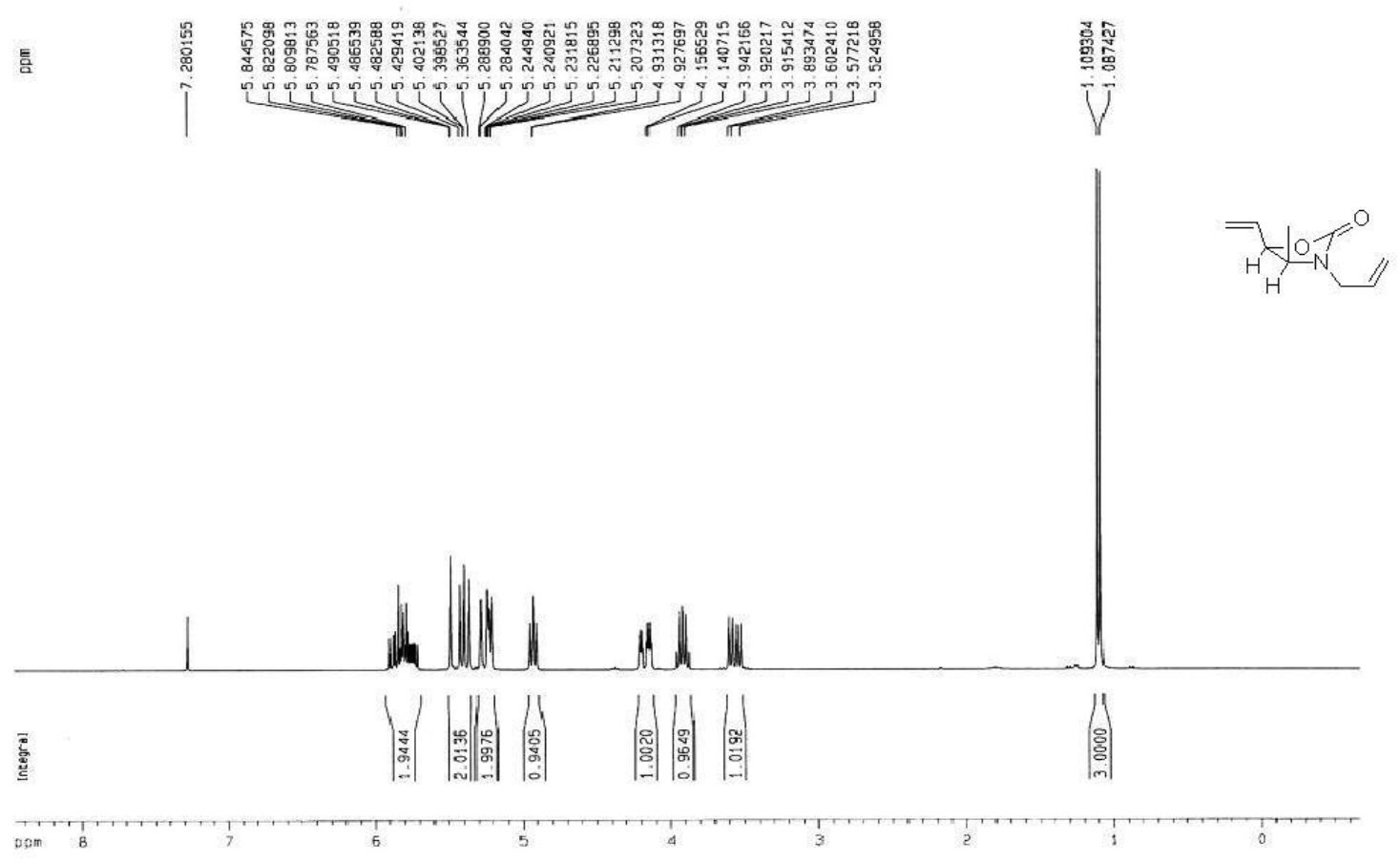

Spectra 15. ${ }^{1} \mathrm{H}$ NMR $\left(300 \mathrm{MHz}, \mathrm{CDCl}_{3}\right)$ of $(4 S, 5 R)$-3-allyl-4-methyl-5-vinyloxazolidin-2-one (11)

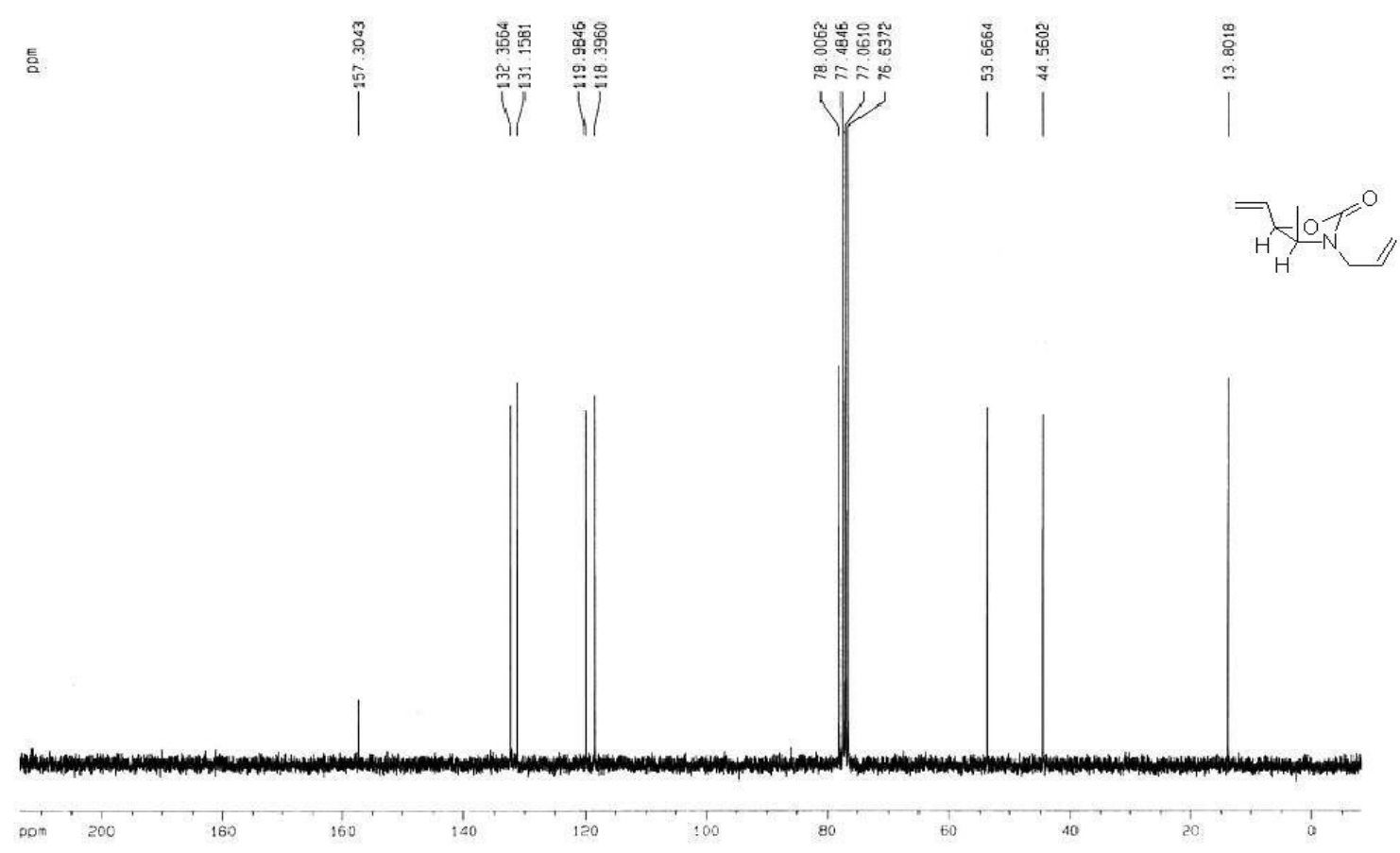

Spectra $16 .{ }^{13} \mathrm{C}$ NMR $\left(300 \mathrm{MHz}, \mathrm{CDCl}_{3}\right)$ of $(4 S, 5 R)$-3-allyl-4-methyl-5-vinyloxazolidin-2-one (11) 


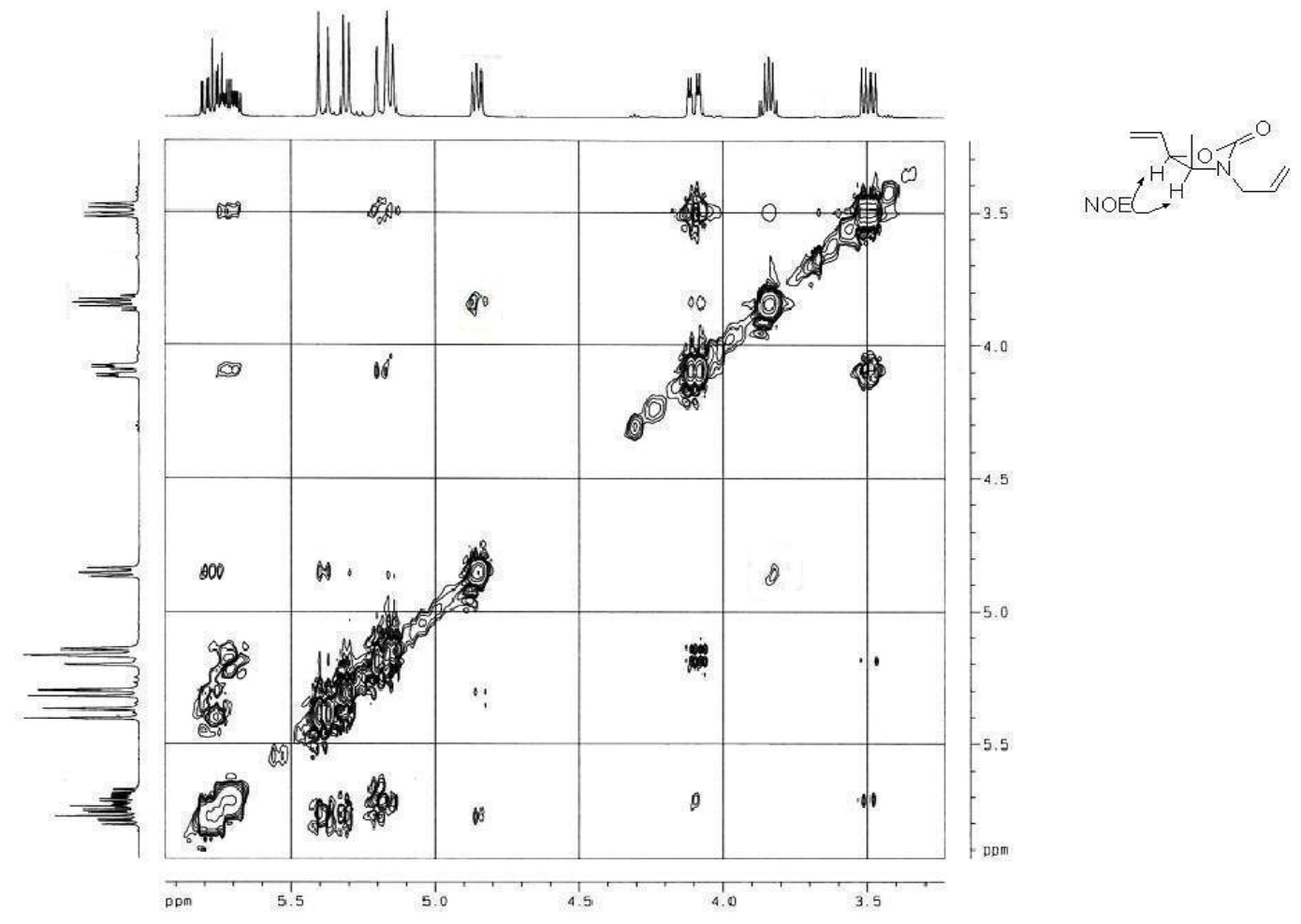

Spectra 17. NOESY (300 MHz, $\left.\mathrm{CDCl}_{3}\right)$ of (4S,5R)-3-allyl-4-methyl-5-vinyloxazolidin-2-one (11)

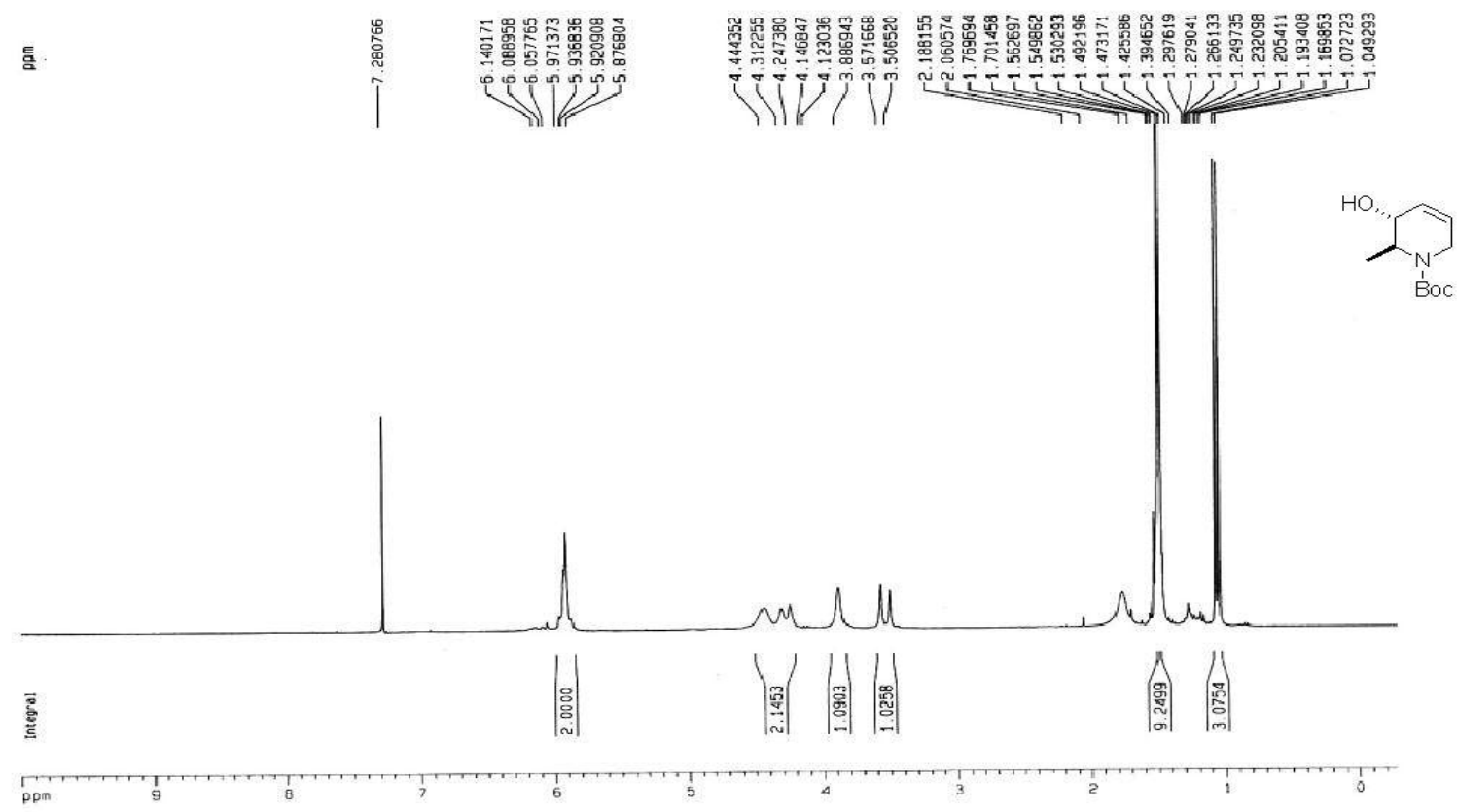

Spectra 18. ${ }^{1} \mathrm{H}$ NMR (300 MHz, $\left.\mathrm{CDCl}_{3}\right)$ of (5R,6S)-tert-butyl-5-hydroxy-6-methyl-5,6dihydropyridine-1(2H)-carbamate (13) 


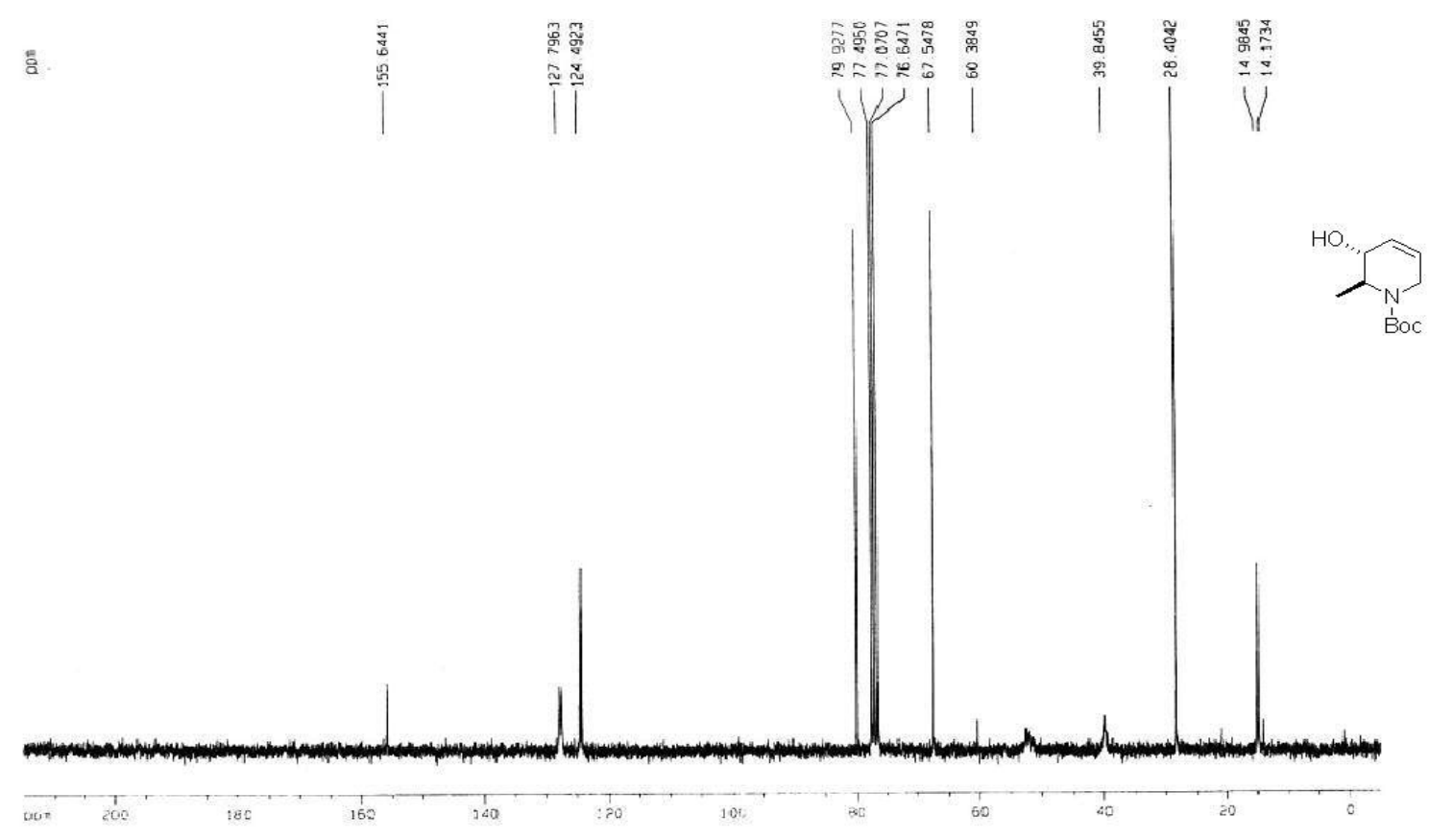

Spectra 19. ${ }^{13} \mathrm{C} \mathrm{NMR}\left(300 \mathrm{MHz}, \mathrm{CDCl}_{3}\right)$ of $(5 R, 6 S)$-tert-butyl 5-hydroxy-6-methyl-5,6dihydropyridine-1(2H)-carbamate (13)

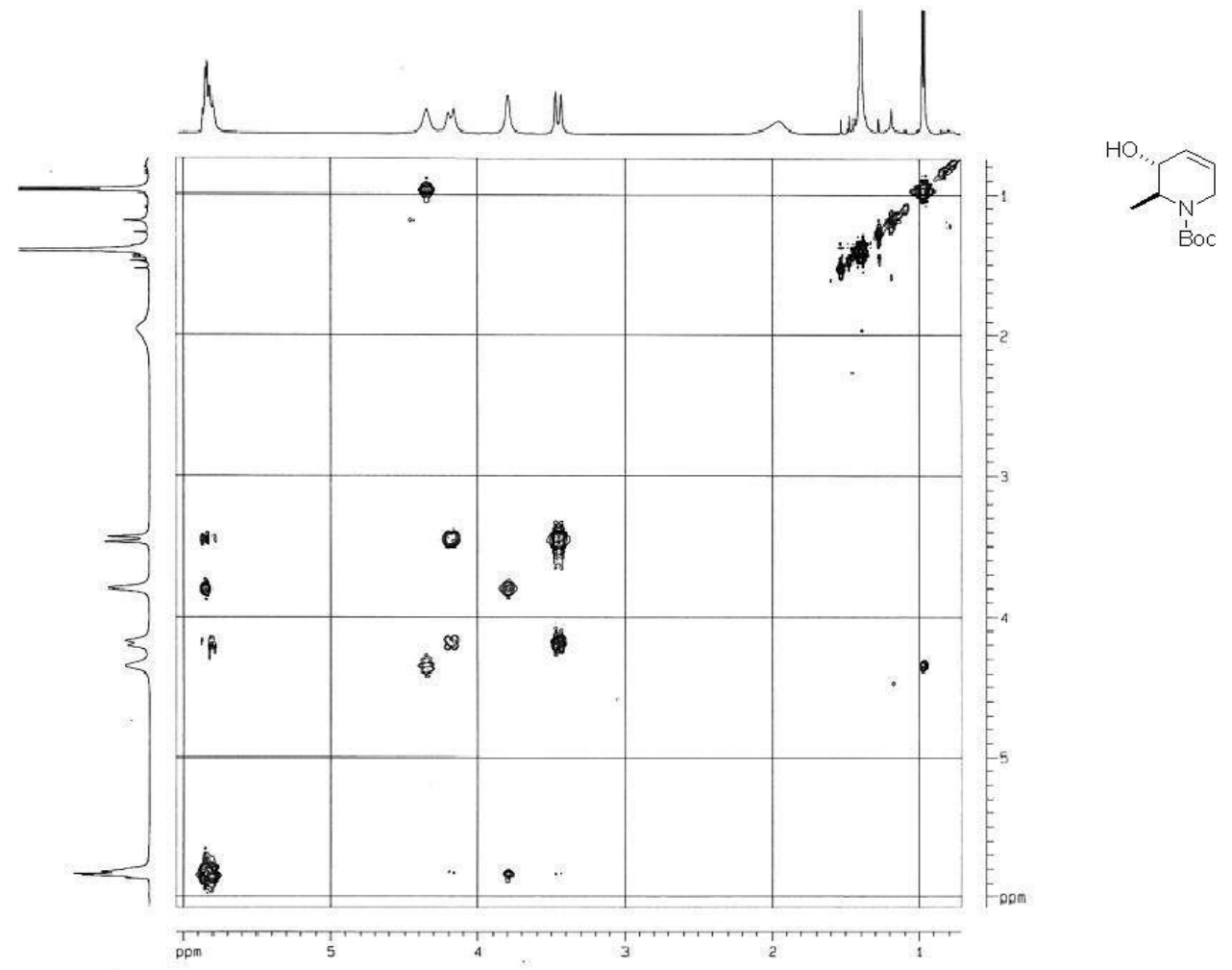

Spectra 20. HOMO COESY $\left(300 \mathrm{MHz} \mathrm{CDCl}_{3}\right)$ of $(5 R, 6 S)$-tert-butyl 5-hydroxy-6-methyl-5,6dihydropyridine-1(2H)-carbamate (13) 


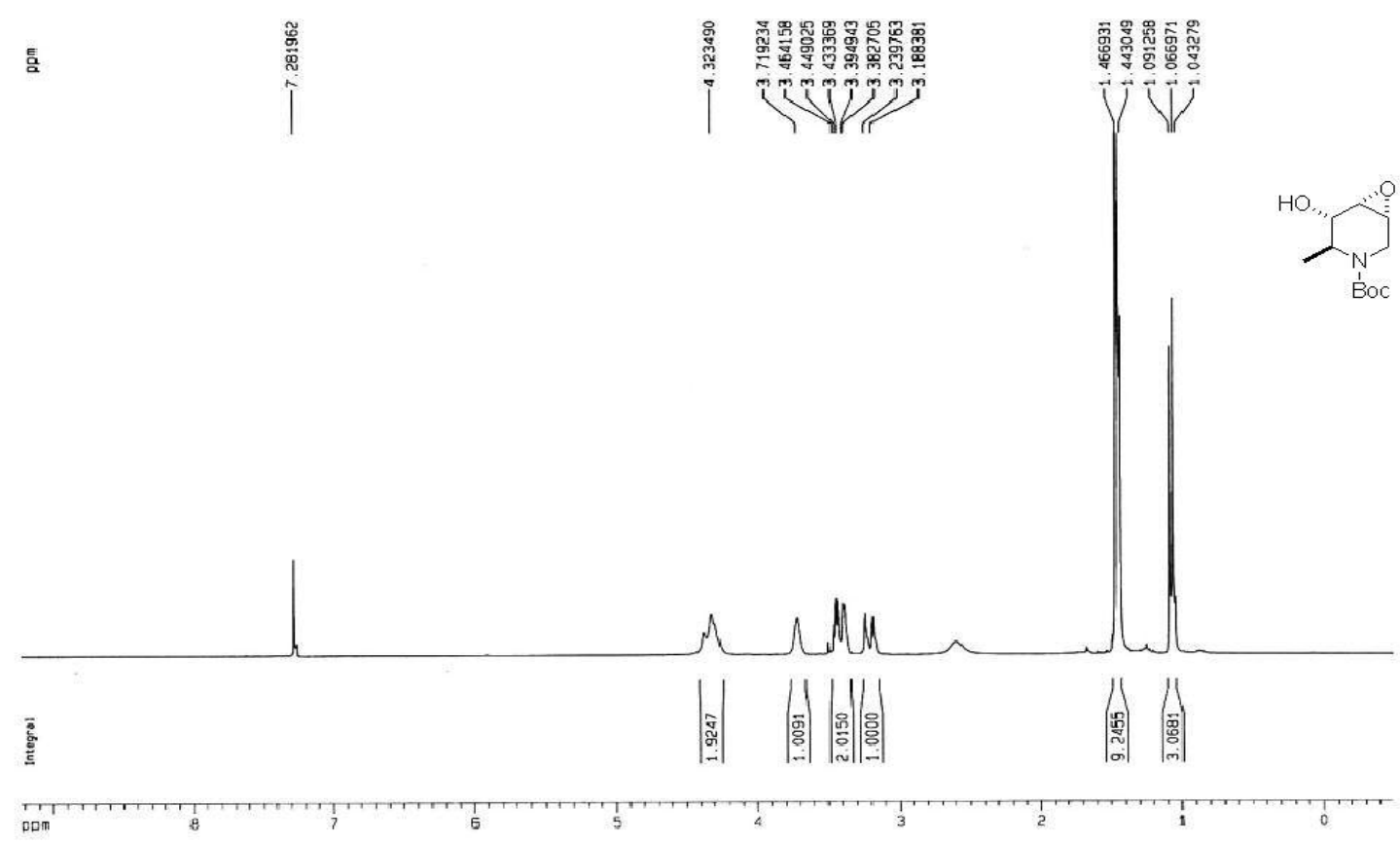

Spectra 21. ${ }^{1} \mathrm{H}$ NMR (300 $\left.\mathrm{MHz} \mathrm{CDCl}_{3}\right)$ of $(1 R, 4 S, 5 S, 6 S)$-tert-butyl-5-hydroxy-4-methyl-7-oxa-3aza-bicyclo[4.1.0]heptane-3-carbamate (15)

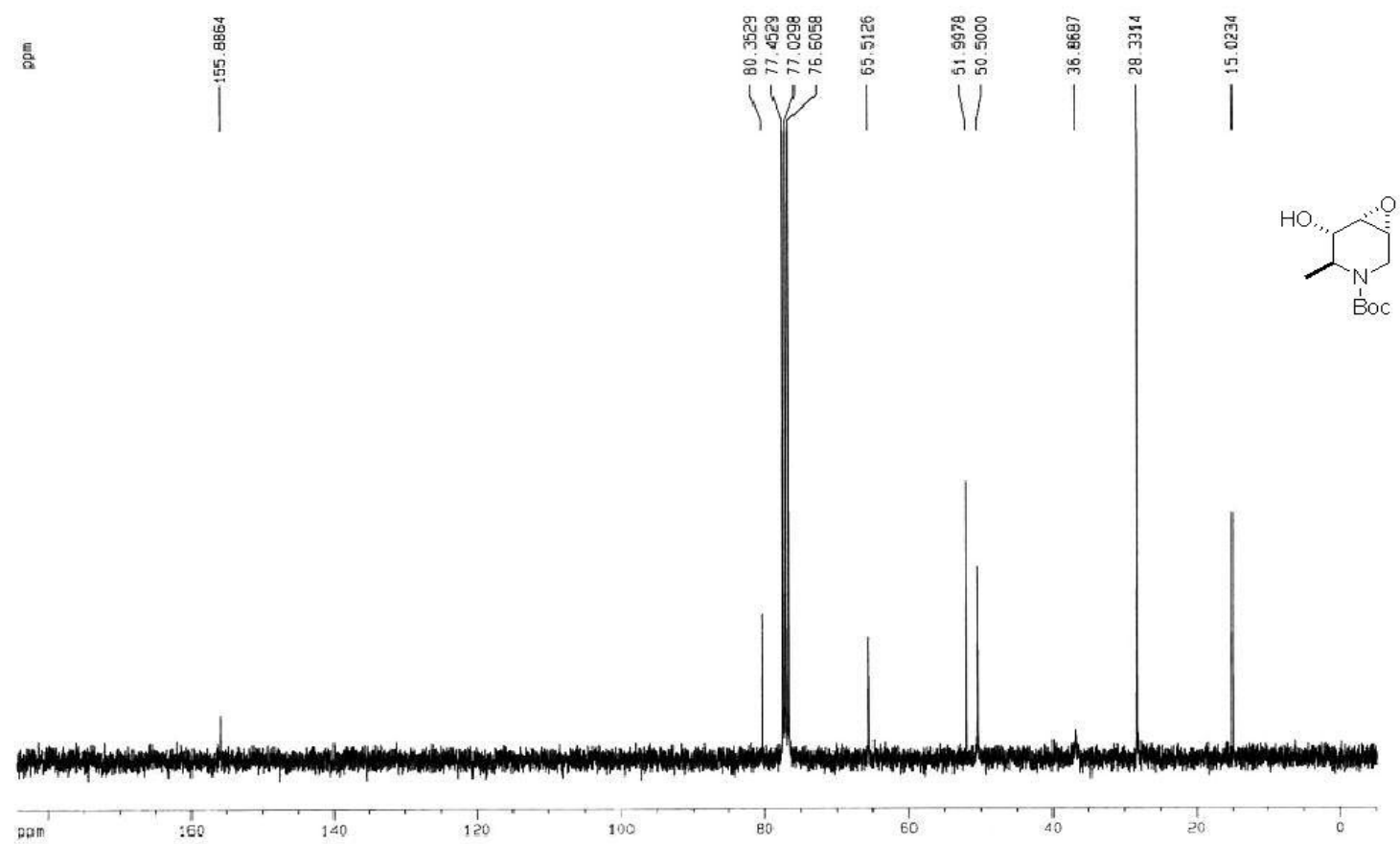

Spectra 22. ${ }^{13} \mathrm{C}$ NMR (300 $\left.\mathrm{MHz} \mathrm{CDCl}_{3}\right)$ of $(1 R, 4 S, 5 S, 6 S)$-tert-butyl-5-hydroxy-4-methyl-7-oxa-3aza-bicyclo[4.1.0]heptane-3-carbamate (15) 


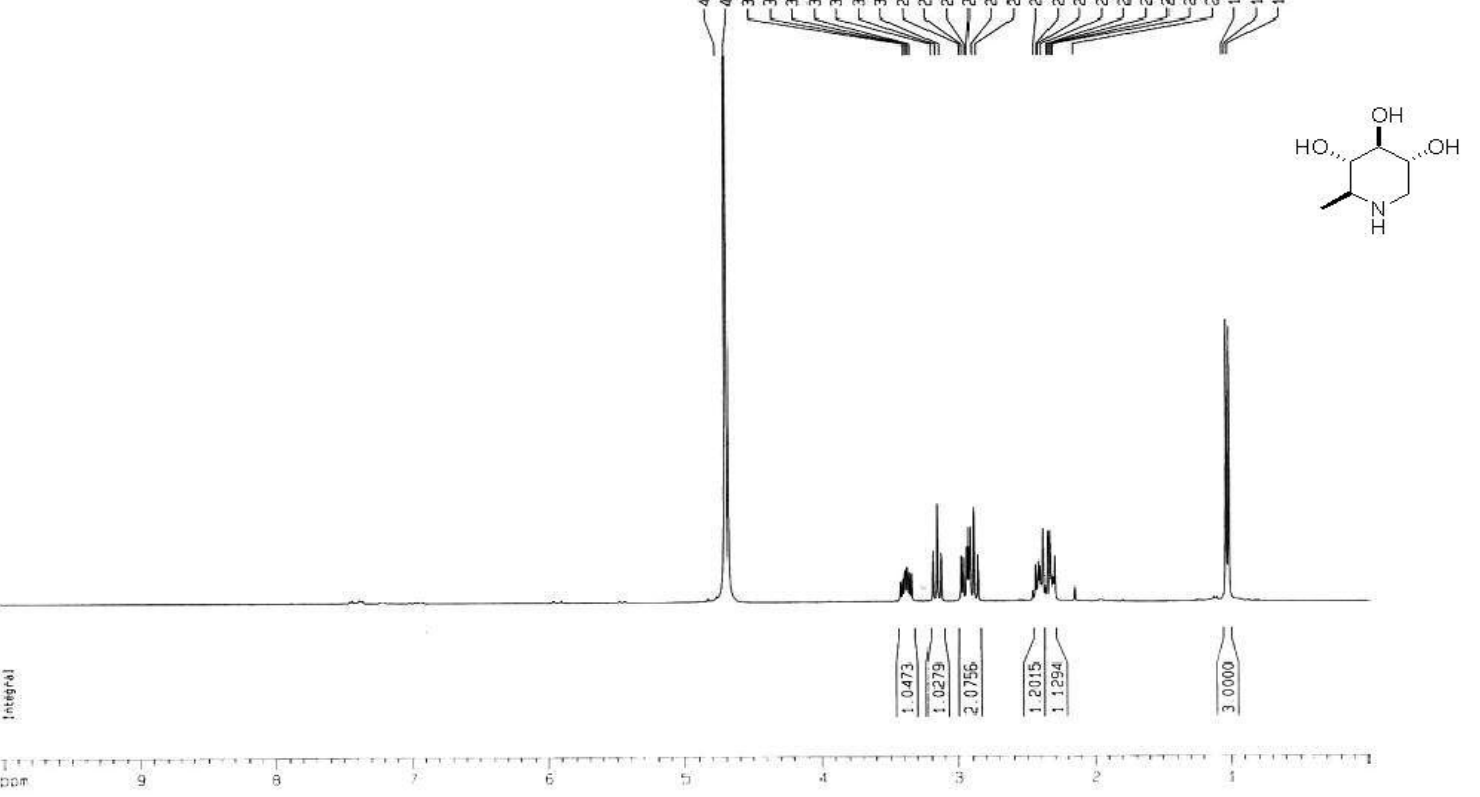

Spectra 23. ${ }^{1} \mathrm{H}$ NMR (300 MHz, $\left.\mathrm{D}_{2} \mathrm{O}\right)$ of $(2 S, 3 S, 4 S, 5 R)$-2-methylpiperidine-3,4,5-triol (1)

톰
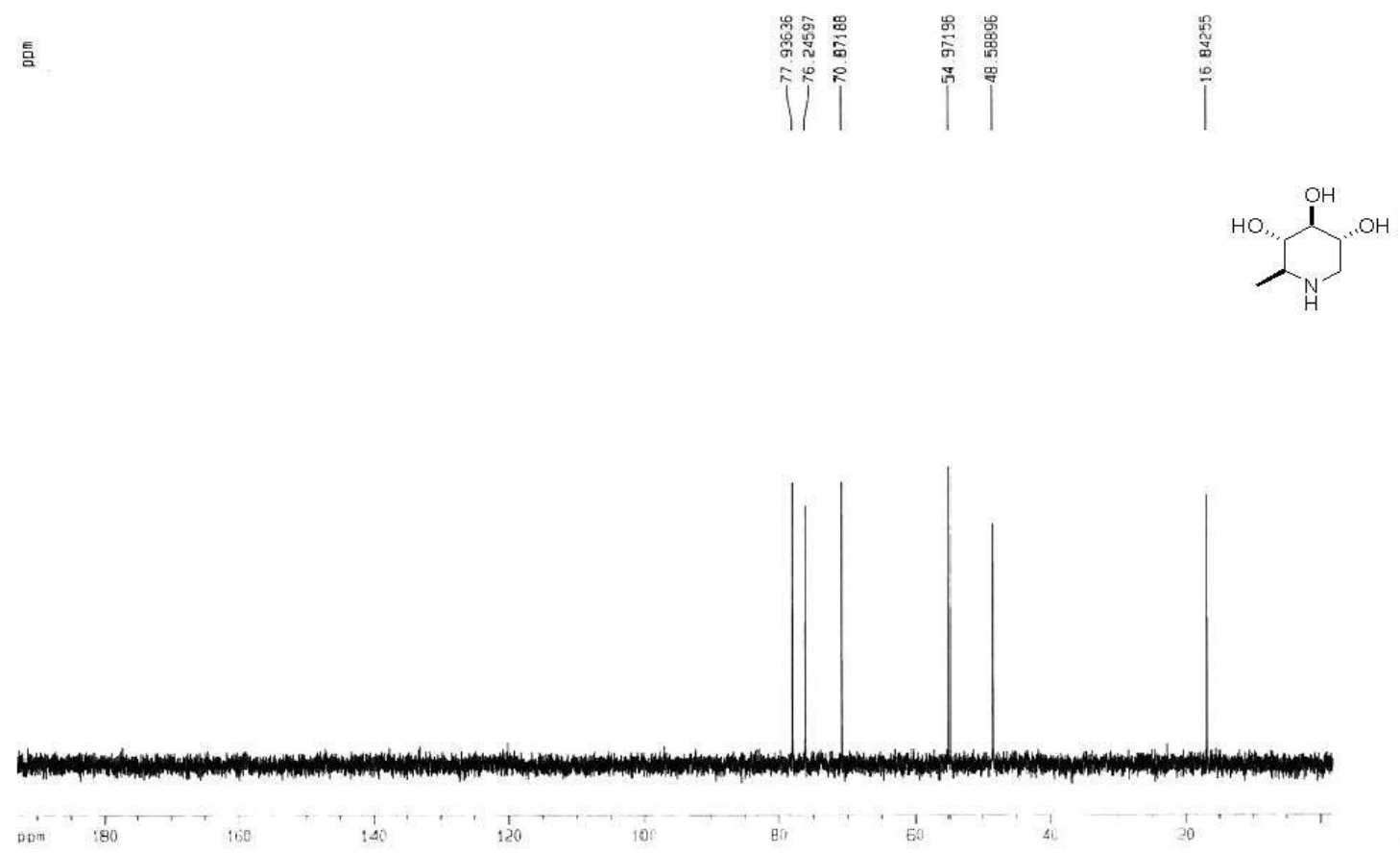

Spectra 24. ${ }^{13} \mathrm{C}$ NMR (300 MHz, $\left.\mathrm{D}_{2} \mathrm{O}\right)$ of $(2 S, 3 S, 4 S, 5 R)-2-$ methylpiperidine-3,4,5-triol (1) 


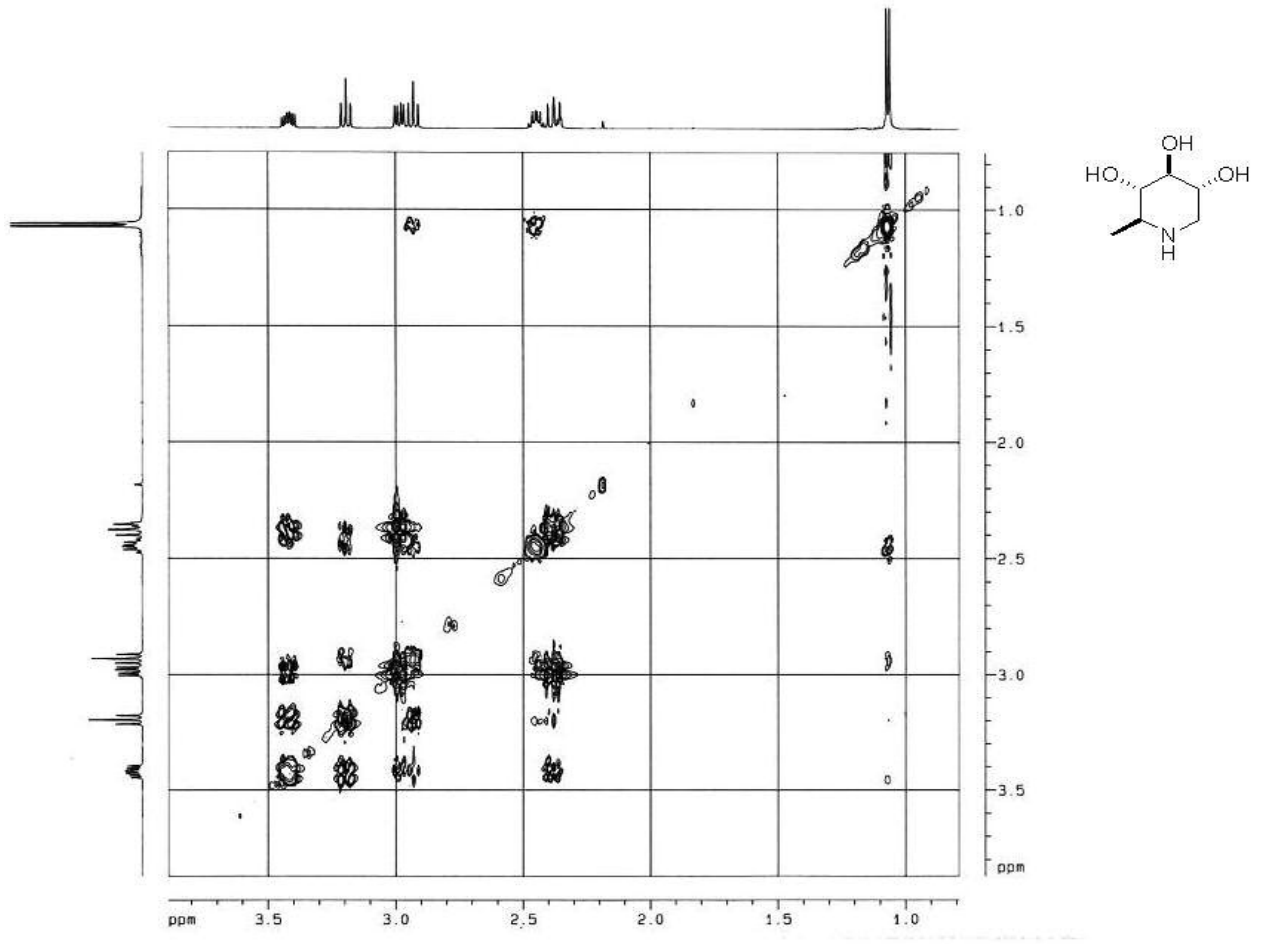

Spectra 25. NOESY (300 MHz, $\left.\mathrm{D}_{2} \mathrm{O}\right)$ of $(2 S, 3 S, 4 S, 5 R)-2$-methylpiperidine-3,4,5-triol (1)

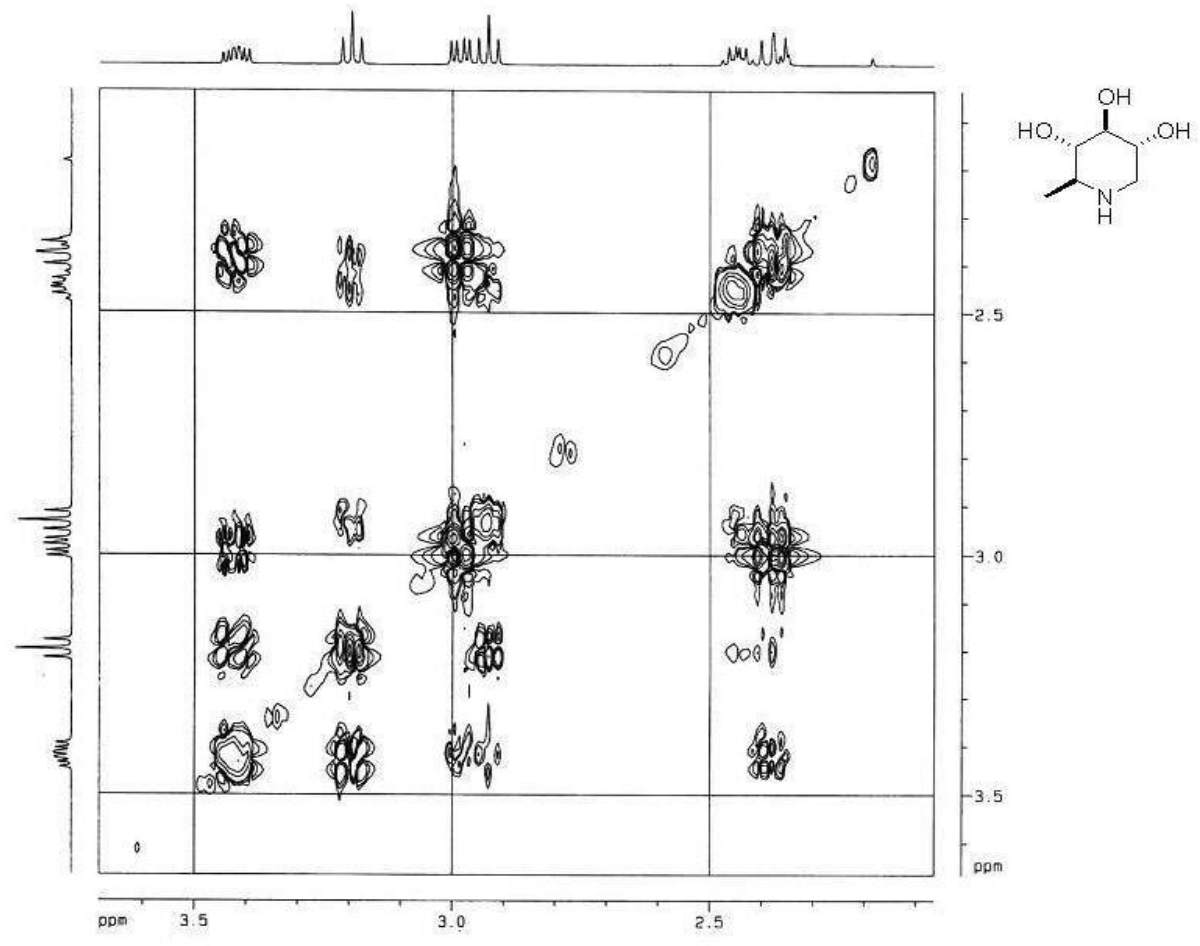

Spectra 26. Expanded NOESY (300 MHz, $\left.\mathrm{D}_{2} \mathrm{O}\right)$ of $(2 S, 3 S, 4 S, 5 R)-2$-methylpiperidine-3,4,5-triol (1) 


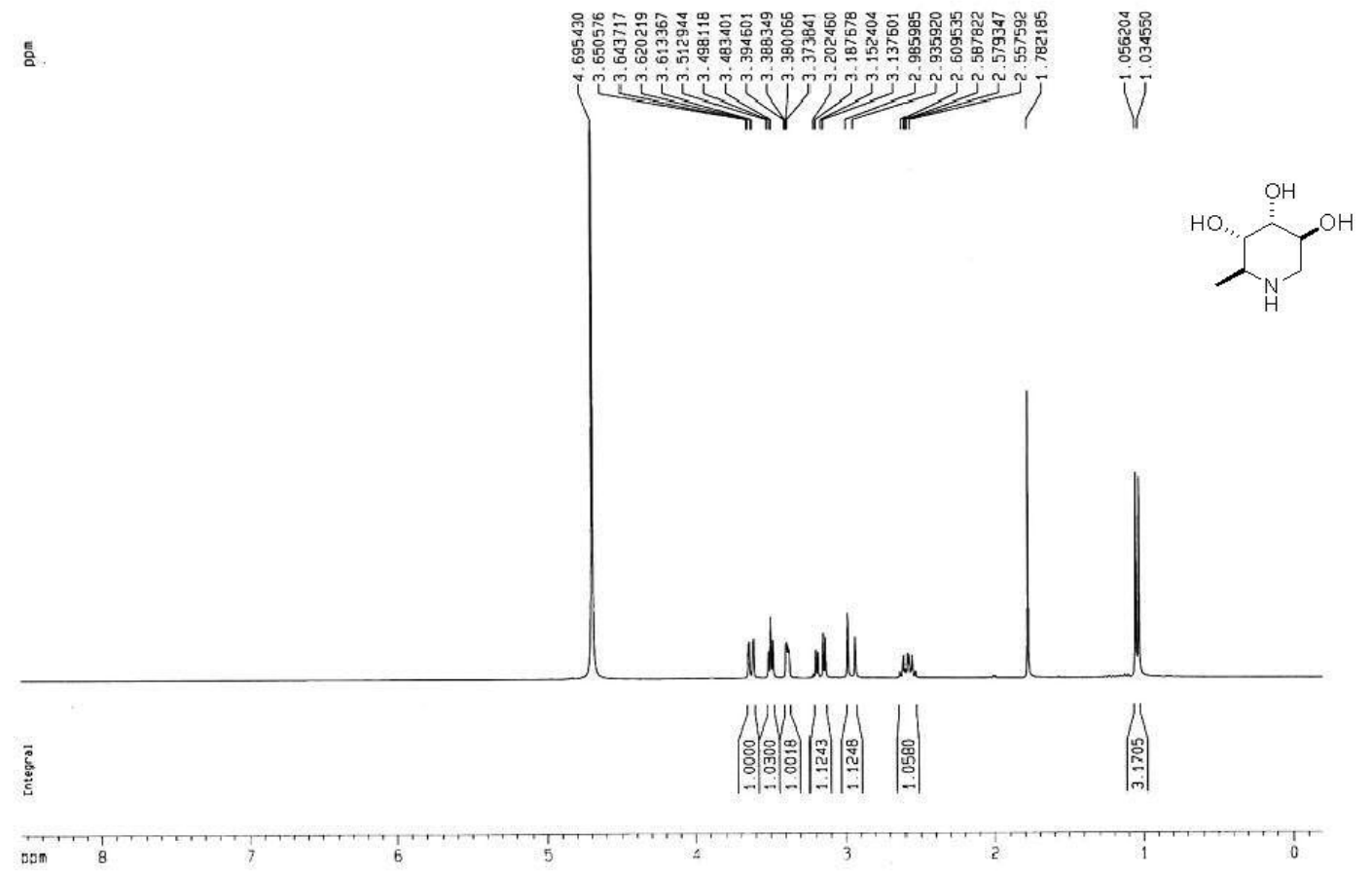

Spectra $27 .{ }^{1} \mathrm{H}$ NMR $\left(300 \mathrm{MHz}, \mathrm{D}_{2} \mathrm{O}\right)$ of $(2 S, 3 S, 4 R, 5 S)$-2-methylpiperidine-3,4,5-triol (2)

ล
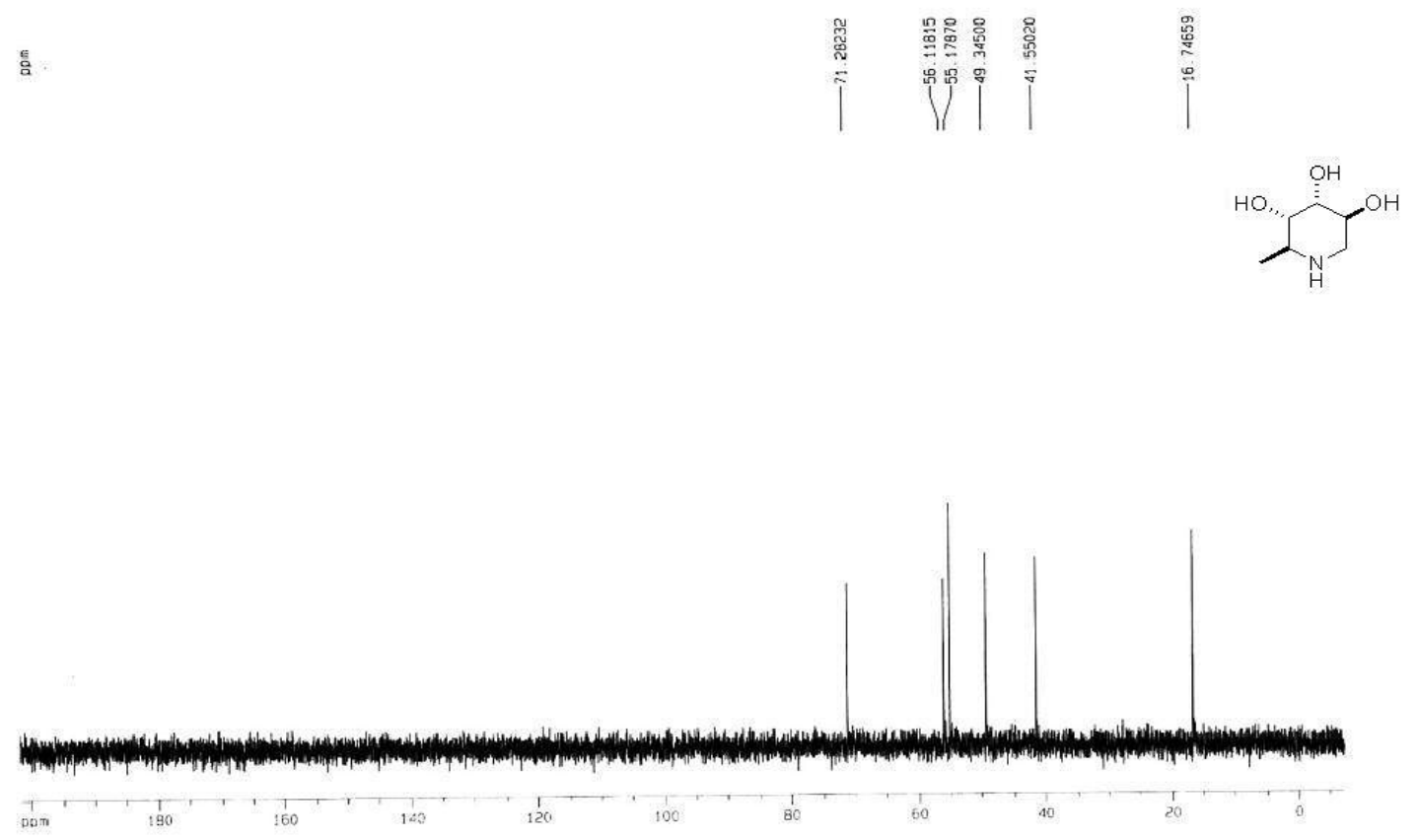

Spectra $28 .{ }^{13} \mathrm{C}$ NMR (300 MHz, $\left.\mathrm{D}_{2} \mathrm{O}\right)$ of $(2 S, 3 S, 4 R, 5 S)$-2-methylpiperidine-3,4,5-triol (2) 


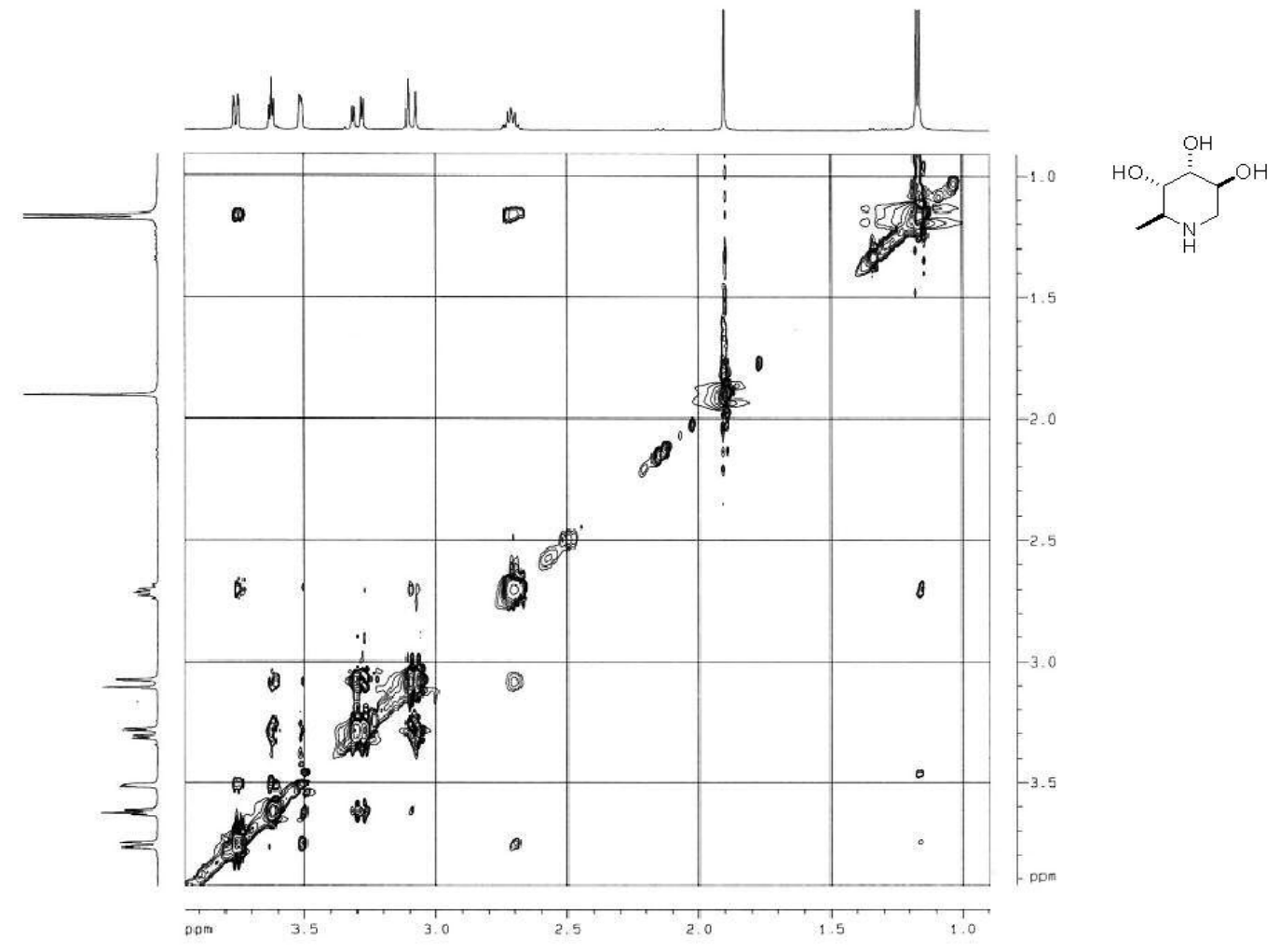

Spectra 29. NOESY (300 MHz, $\left.\mathrm{D}_{2} \mathrm{O}\right)$ of $(2 S, 3 S, 4 R, 5 S)$-2-methylpiperidine-3,4,5-triol (2)

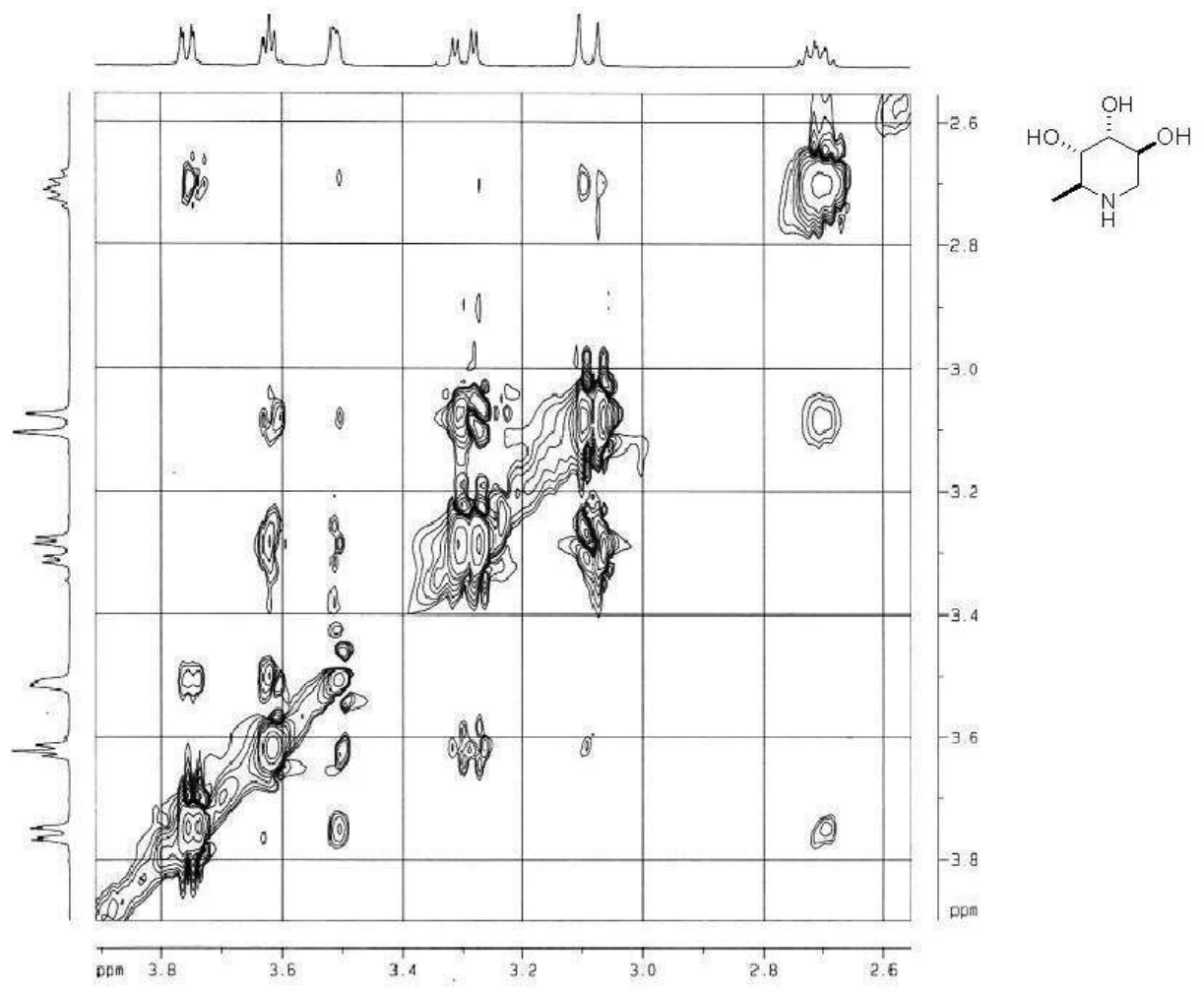

Spectra 30. Expanded NOESY $\left(300 \mathrm{MHz}, \mathrm{D}_{2} \mathrm{O}\right)$ of $(2 S, 3 S, 4 R, 5 S)-2$-methylpiperidine-3,4,5-triol (2) 


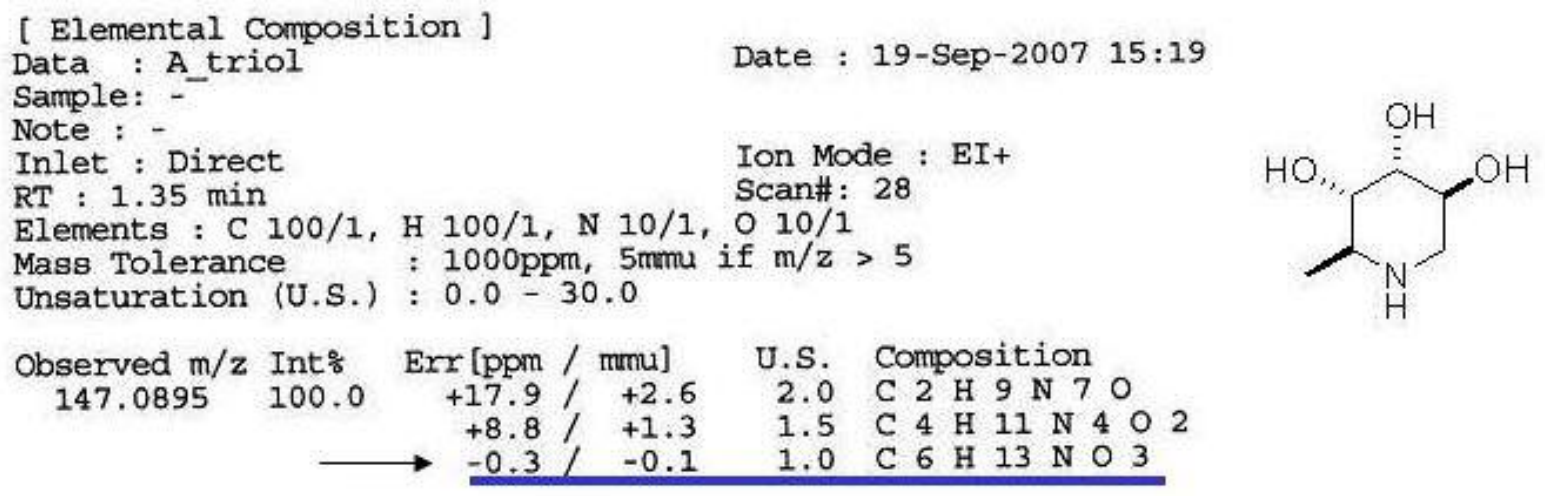

\section{Spectra 31. HR mass of $(2 S, 3 S, 4 R, 5 S)-2$-methylpiperidine-3,4,5-triol (2)}

高

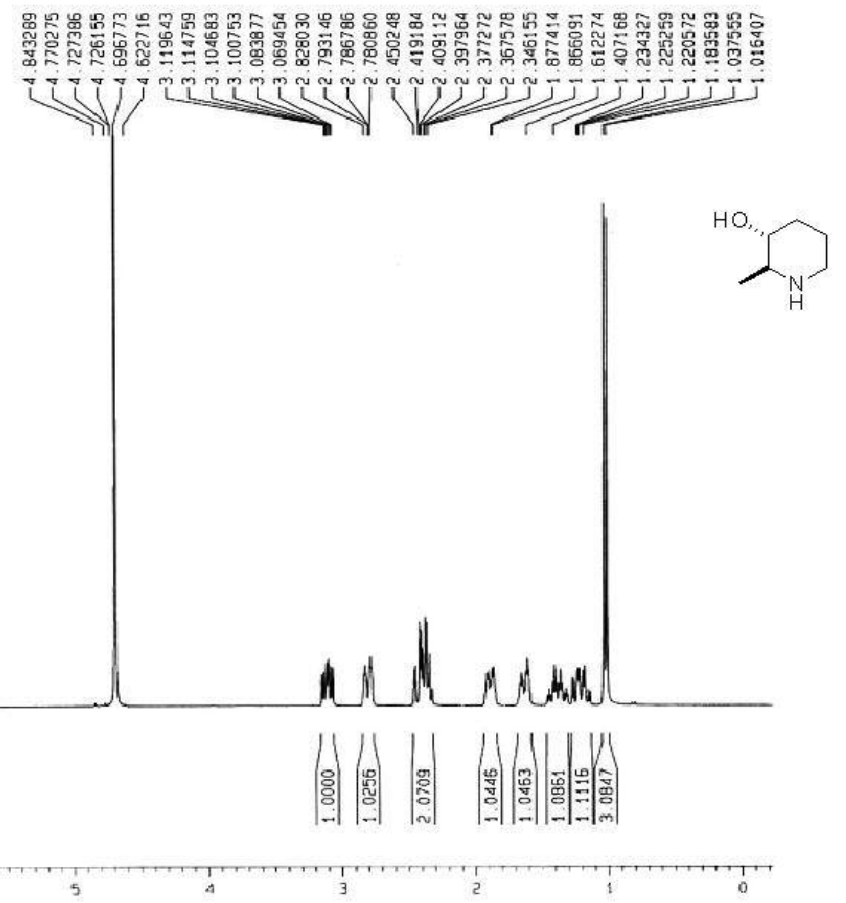

Spectra 32. ${ }^{1} \mathrm{H}$ NMR (300 MHz, $\left.\mathrm{D}_{2} \mathrm{O}\right)$ of (2S,3R)-2-methylpiperidin-3-ol (3) 


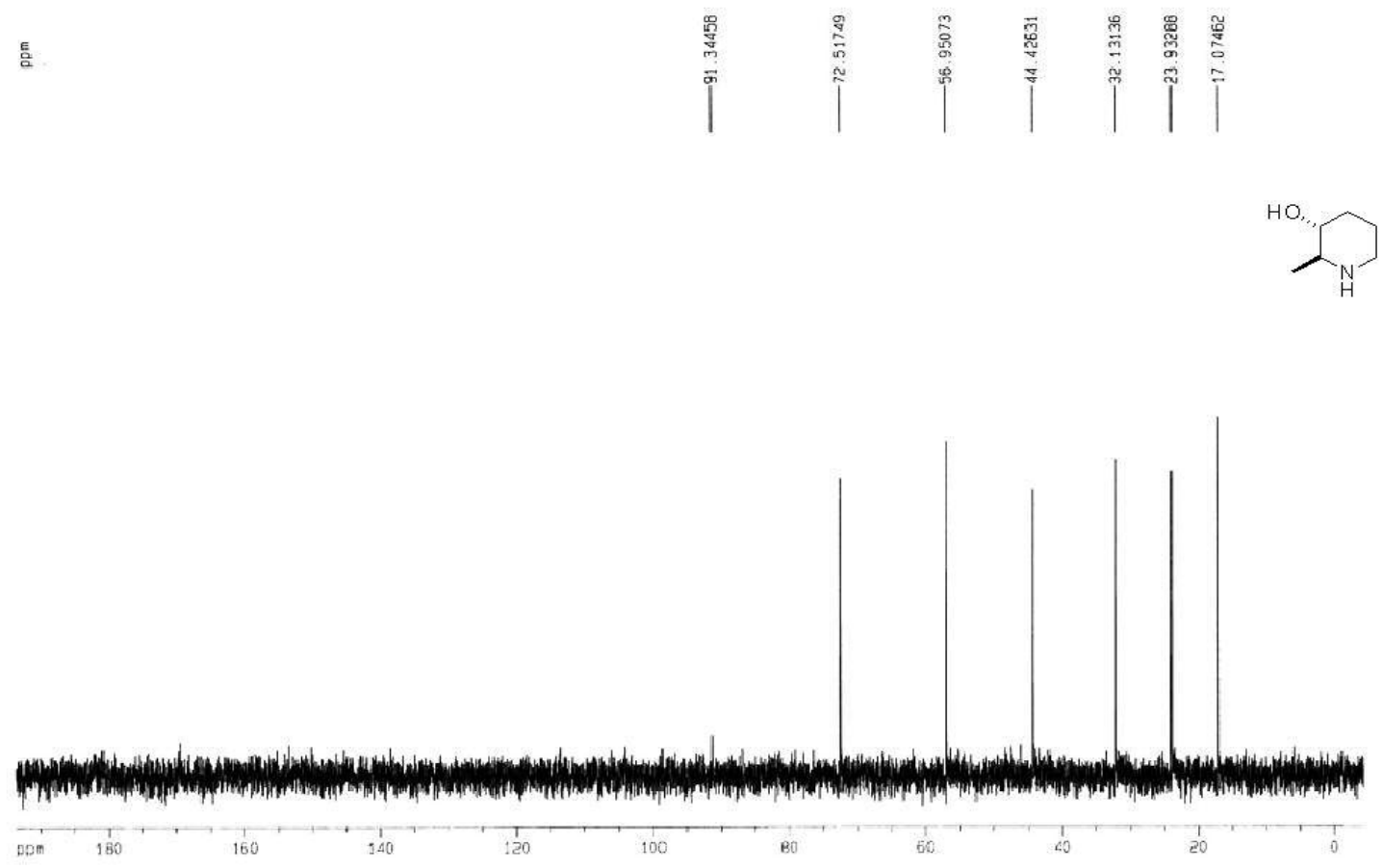

Spectra 33. ${ }^{13} \mathrm{C}$ NMR (300 MHz, $\left.\mathrm{D}_{2} \mathrm{O}\right)$ of (2S,3R)-2-methylpiperidin-3-ol (3)

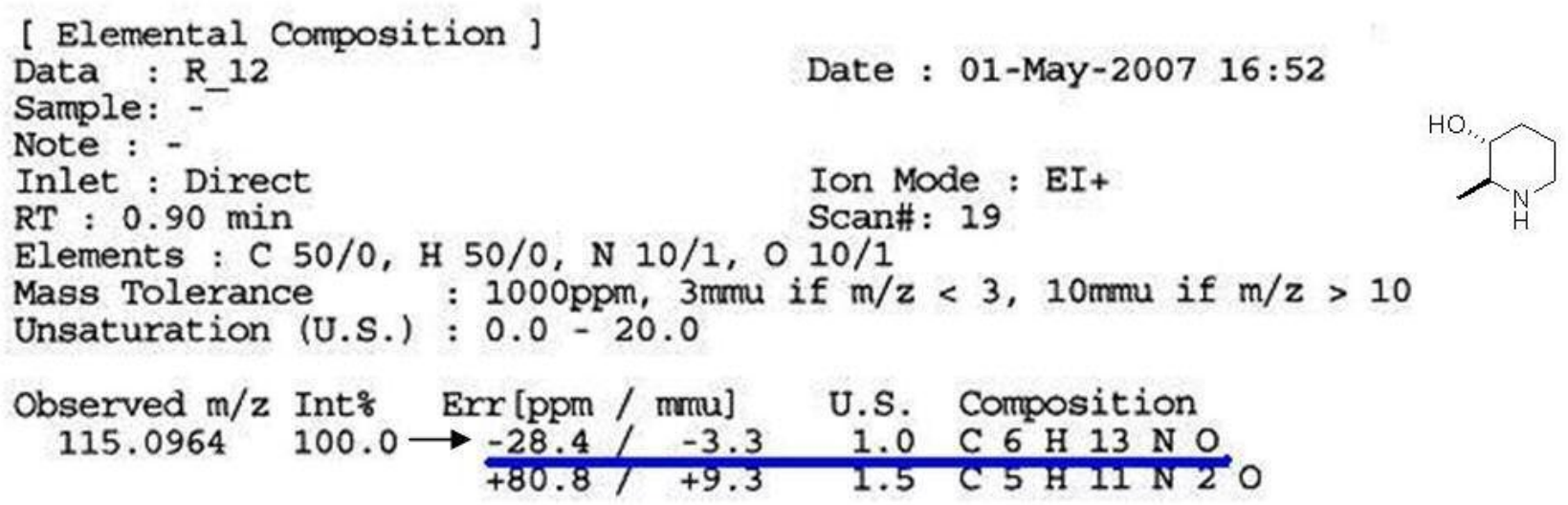

Spectra 34. HR mass of (2S,3R)-2-methylpiperidin-3-ol (3) 


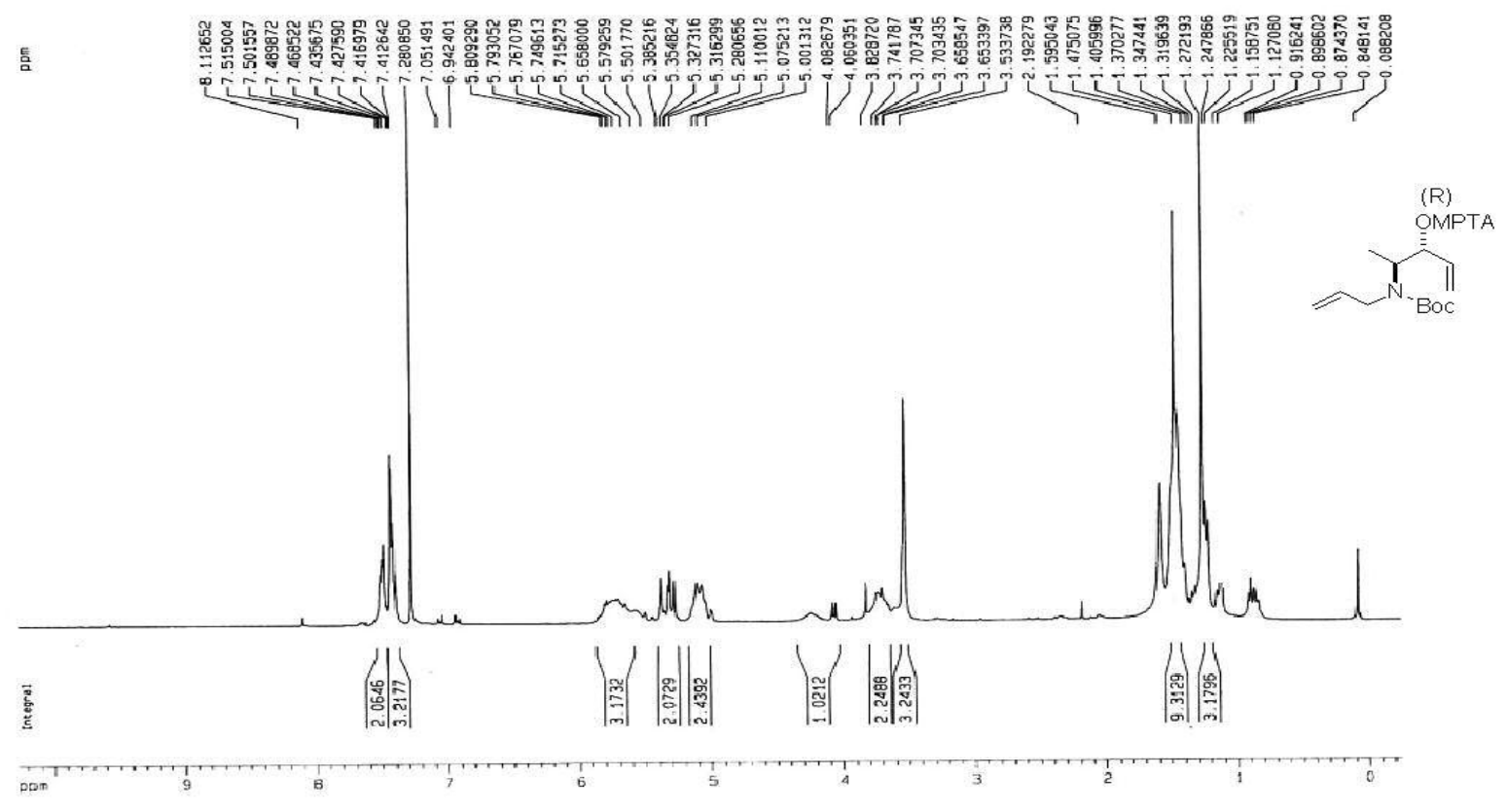

Spectra 35. ${ }^{1} \mathrm{H}$ NMR $\left(300 \mathrm{MHz}, \mathrm{CDCl}_{3}\right)$ of $(3 R, 4 S)$-4-[allyl(tert-butoxycarbonyl)amino]pent-1-en3-yl 3,3,3-trifluoro-2-methoxy-2-phenylpropanoate (12)

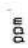
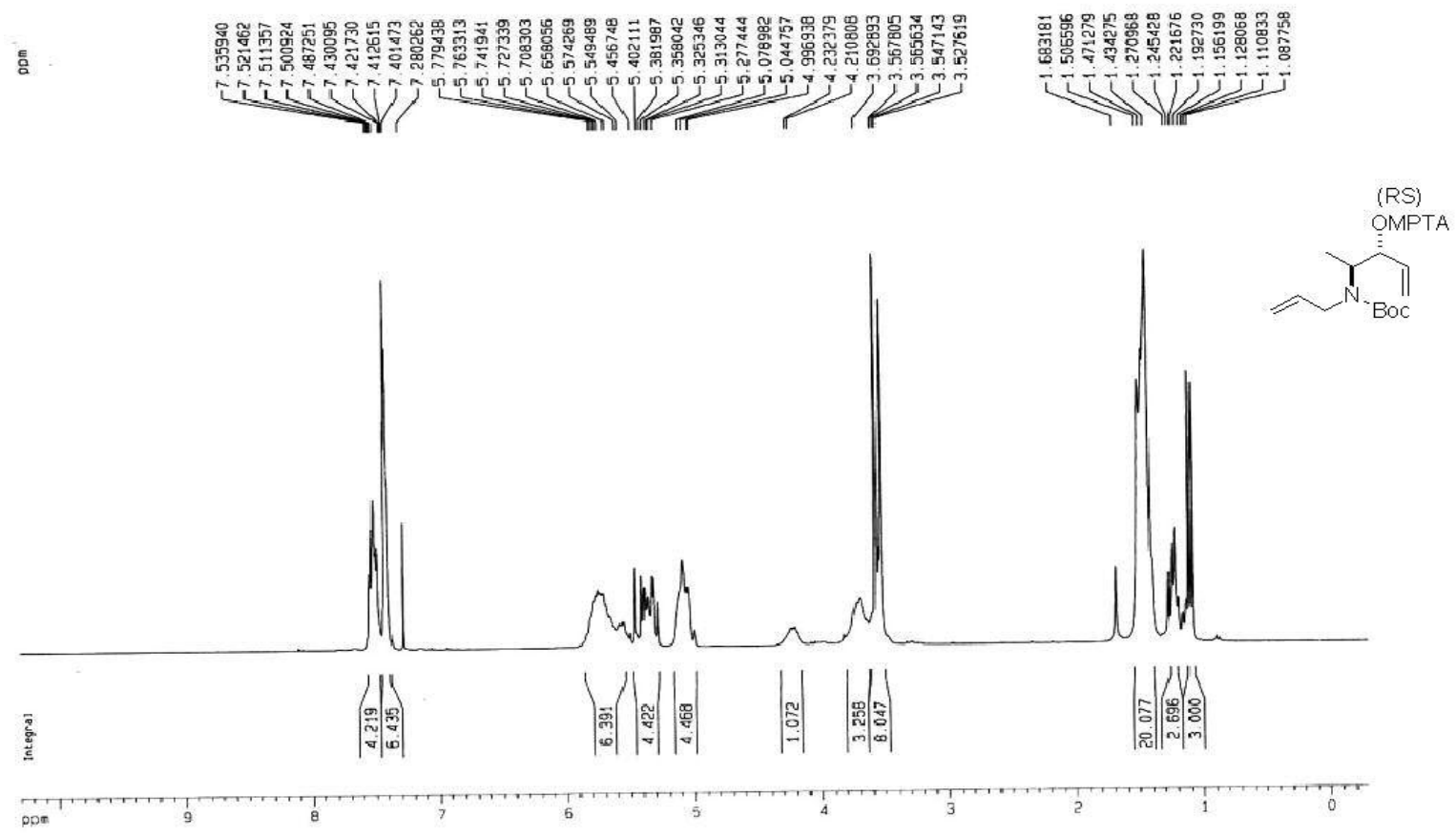

Spectra 36. ${ }^{1} \mathrm{H}$ NMR $\left(300 \mathrm{MHz}, \mathrm{CDCl}_{3}\right)$ of $(3 R, 4 S)-4$-[allyl(tert-butoxycarbonyl)amino]pent-1-en3-yl 3,3,3-trifluoro-2-methoxy-2-phenylpropanoate (RS mixture) (12) 
[ Elemental Composition ]

Data : B_tri_HR

Date : 13-Feb-2008 14:31

Sample: -

Note : -

Inlet : Direct

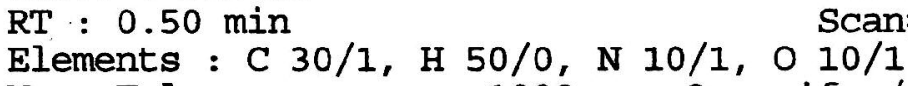

Ion Mode : $\mathrm{FAB}+$

Mass Tolerance

: $1000 \mathrm{ppm}, 3 \mathrm{mmu}$ if $\mathrm{m} / \mathrm{z}<3,10 \mathrm{mmu}$ if $\mathrm{m} / \mathrm{z}>10$

Unsaturation (U.S.) : $-0.5-20.0$

Observed $\mathrm{m} / \mathrm{z}$ Int: Err [ppm / mmu] 148.0970

$$
\begin{aligned}
& 0.3+15.9 /+2.3 \\
& +6.8 /+1.0
\end{aligned}
$$

U.S. Composition

1.5

1.0

C $2 \mathrm{H} 10 \mathrm{~N} 7 \mathrm{O}$

(NOH

Calcd $\left[(\mathrm{M}+\mathrm{H})^{+}\right]: 148.0974$

\section{compound 1}

Spectra 37. FAB-HRMS data of compound 1.

[ Elemental Composition]

Data : 4_DNJ_HR

Date : 13-Feb-2008 13:38

Page: 1

Sample: -

Note : -

Inlet : Direct

$\mathrm{RT}: 0.17 \mathrm{~min}$

Ion Mode : FAB+

Scan\# : 3

Elements : C 14/10, H 32/0, N 10/1, O 10/1, Si 5/1

Mass Tolerance : $1000 \mathrm{ppm}, 3 \mathrm{mmu}$ if $\mathrm{m} / \mathrm{z}<3,100 \mathrm{mmu}$ if $\mathrm{m} / \mathrm{z}>100$

Unsaturation (U.S.) : $-0.5-10.0$

Observed m/z Int $\frac{1}{6}$ Err [ppm / mmu] U.S. Composition

$\begin{array}{lllllll}290.2832 & 19.8+234.4 /+68.1 & 0.5 & \text { C } 14 \mathrm{H} 32 \mathrm{~N} & 0 & 3 \mathrm{Si}\end{array}$

$\overbrace{\mathrm{NHBoc}}^{\text {OTBS }}$ Calcd $\left[(\mathrm{M}+\mathrm{H})^{+}\right]: 290.2151$

compound 6

Spectra 38. FAB-HRMS data of compound 6. 


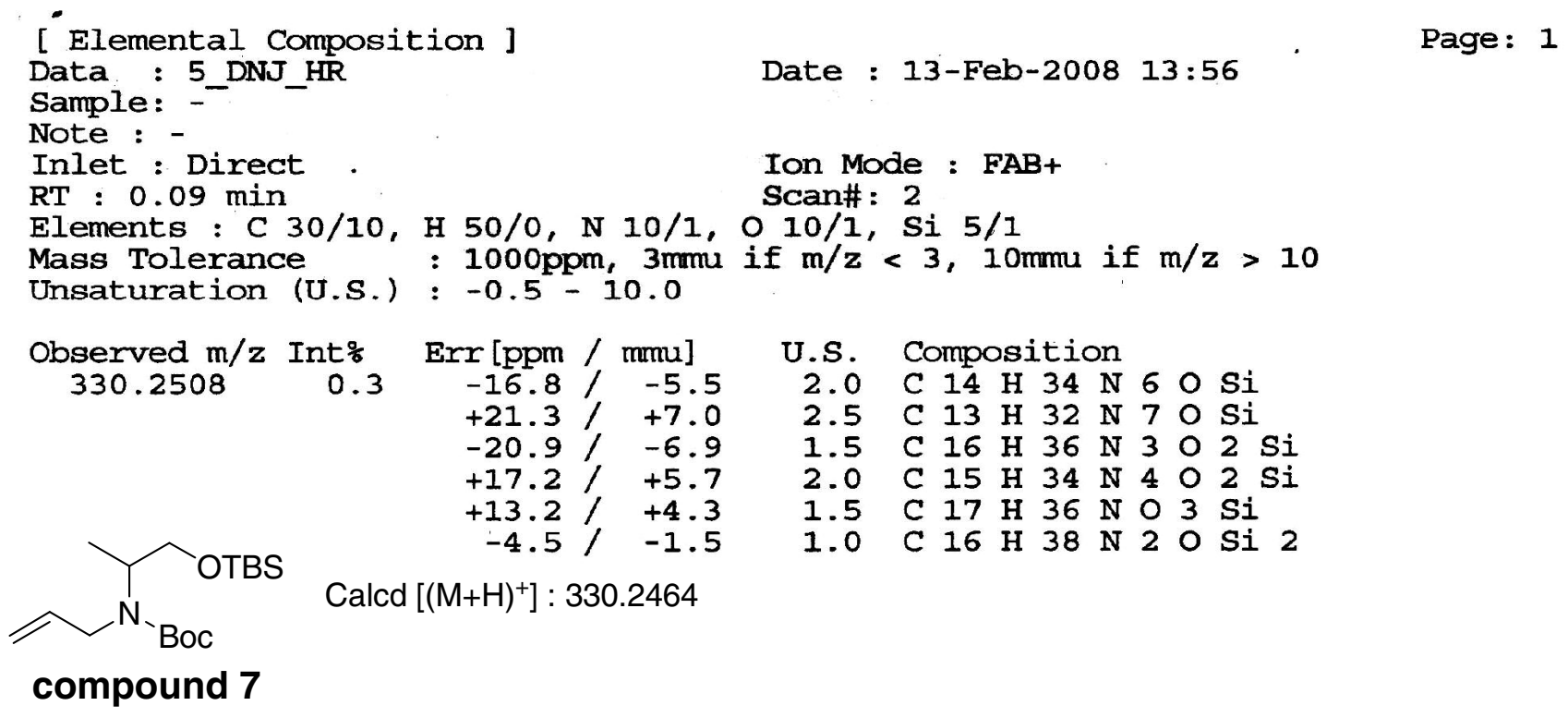

Spectra 39. FAB-HRMS data of compound 7.

[ Elemental Composition ]

Data : 6_DNJ_HR

Date : 13-Feb-2008 $14: 11$<smiles>C=CCN(C(=O)OC(C)(C)C)C(C)C=O</smiles>

\section{compound 9}

Spectra 40. FAB-HRMS data of compound 9. 
[ Elemental Composition ]

Data : HFAB-POS-080131003

Date : 31-Jan-2008 15:20

Page: 1

Sample: A-7a

Note : with PEG400

Inlet : Direct

$\mathrm{RT}: 4.07 \mathrm{~min}$

Elements : C $13 / 0, \mathrm{H} 24 / 0, \mathrm{O} 3 / 0, \mathrm{~N} 1 / 0$

Mass Tolerance : $5 \mathrm{ppm}, 5 \mathrm{mmu}$ if $\mathrm{m} / \mathrm{z}>1000$

Unsaturation (U.S.) : $0.0-100.0$

Observed $\mathrm{m} / \mathrm{z}$ Int: $\mathrm{Err}[\mathrm{ppm} / \mathrm{mmu}]$ 242.1764 $0.6+3.2 /+0.8$

U.S. Composition

Ion Mode : FAB+

Scan\# : 30

$2.5 \mathrm{C} 13 \mathrm{H} 2403 \mathrm{~N}$<smiles>C=CCN(C(=O)OC(C)(C)C)C(C)C(O)C=C</smiles>

Calcd $\left[(\mathrm{M}+\mathrm{H})^{+}\right]: 242.1756$

compound 10

Spectra 41. FAB-HRMS data of compound 10.

[-Elemental Composition ]

Data : HFAB-POS-080131004

Date : 31-Jan-2008 16:05

Page: 1

Sample: MTPA

Note : with PEG400

Inlet : Direct

RT : $0.33 \mathrm{~min}$

Ion Mode : FAB+

Elements : $\mathrm{C} 23 / 0, \mathrm{H} 31 / 0,05 / 0, \mathrm{~N} 1 / 0$,

Mass Tolerance : 5ppm, $5 \mathrm{mmu}$ if $\mathrm{m} / \mathrm{z}>1000$

Unsaturation (U.S.) : $0.0-100.0$

Observed $\mathrm{m} / \mathrm{z}$ Int\% Err [ppm / mmu U.S. Composition
458.2156
1.5
$+0.4 /+0.2$
$7.5 \mathrm{C} 23 \mathrm{H} 31 \bigcirc 5 \mathrm{NF} 3$<smiles>C=CCN(C(=O)OC(C)(C)C)C(C)C(C=C)OC(C)(C)C</smiles>

Calcd $\left[(\mathrm{M}+\mathrm{H})^{+}\right]: 458.2154$

compound 12

Spectra 42. FAB-HRMS data of compound 12. 